\title{
Supporting Information for Publication Modeling Hydrogen Release from Water with Borane and Alane Catalysts: A Unified Reaction Valley Approach
}

Sadisha Nanayakkara, Marek Freindorf, Yunwen Tao, and Elfi Kraka

Computational and Theoretical Chemistry Group (CATCO),

Department of Chemistry, Southern Methodist University,

3215 Daniel Avenue, Dallas, Texas 75275-0314, United States

ekraka@smu.edu

http://www.smu.edu/catco 


\section{Reaction schemes}

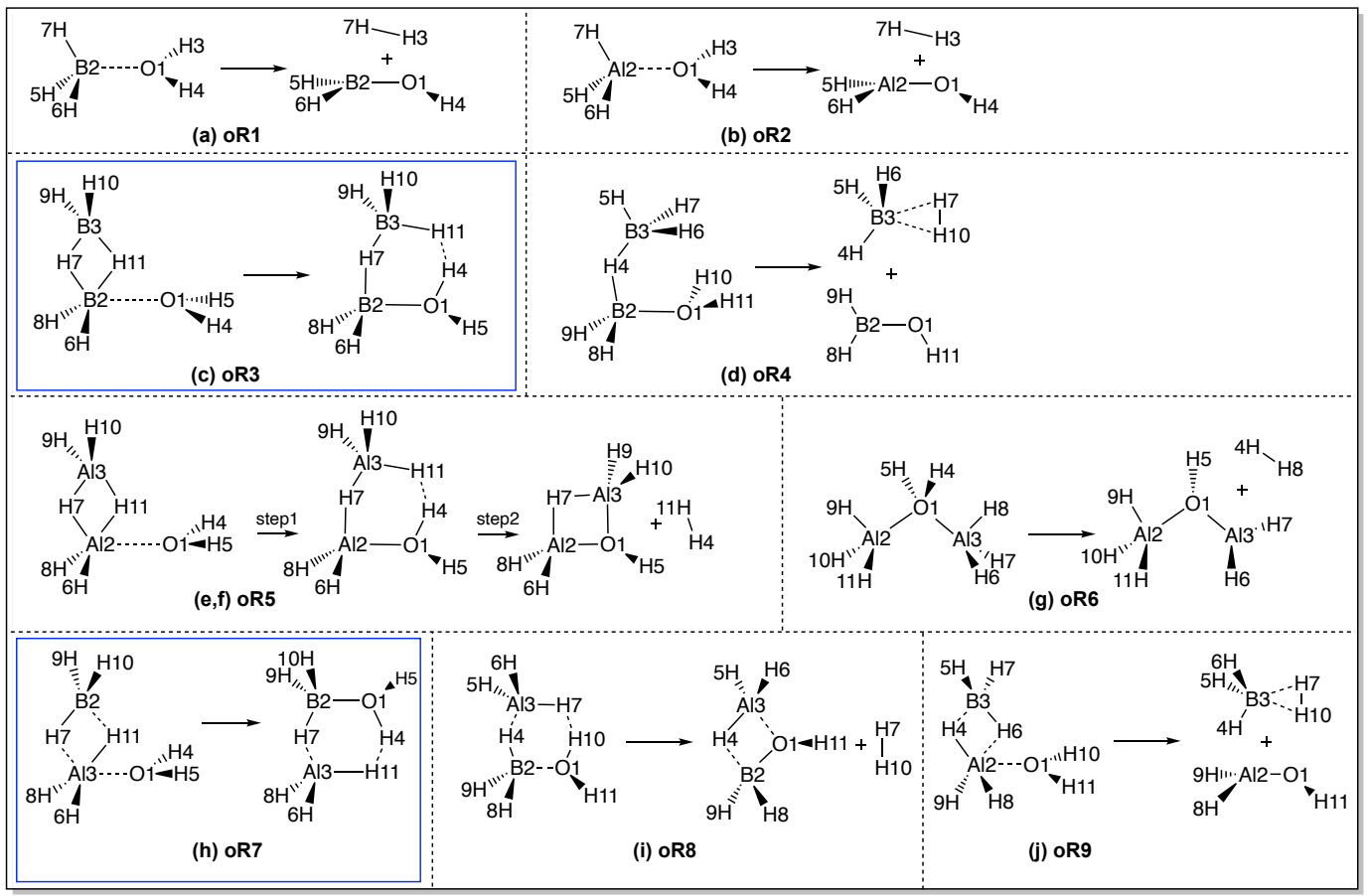

Fig. S1: Reactions oR1 - oR9 with one-water molecule investigated in this work. Reactions shown within blue boxes represent those which do not release hydrogen during the reaction. 


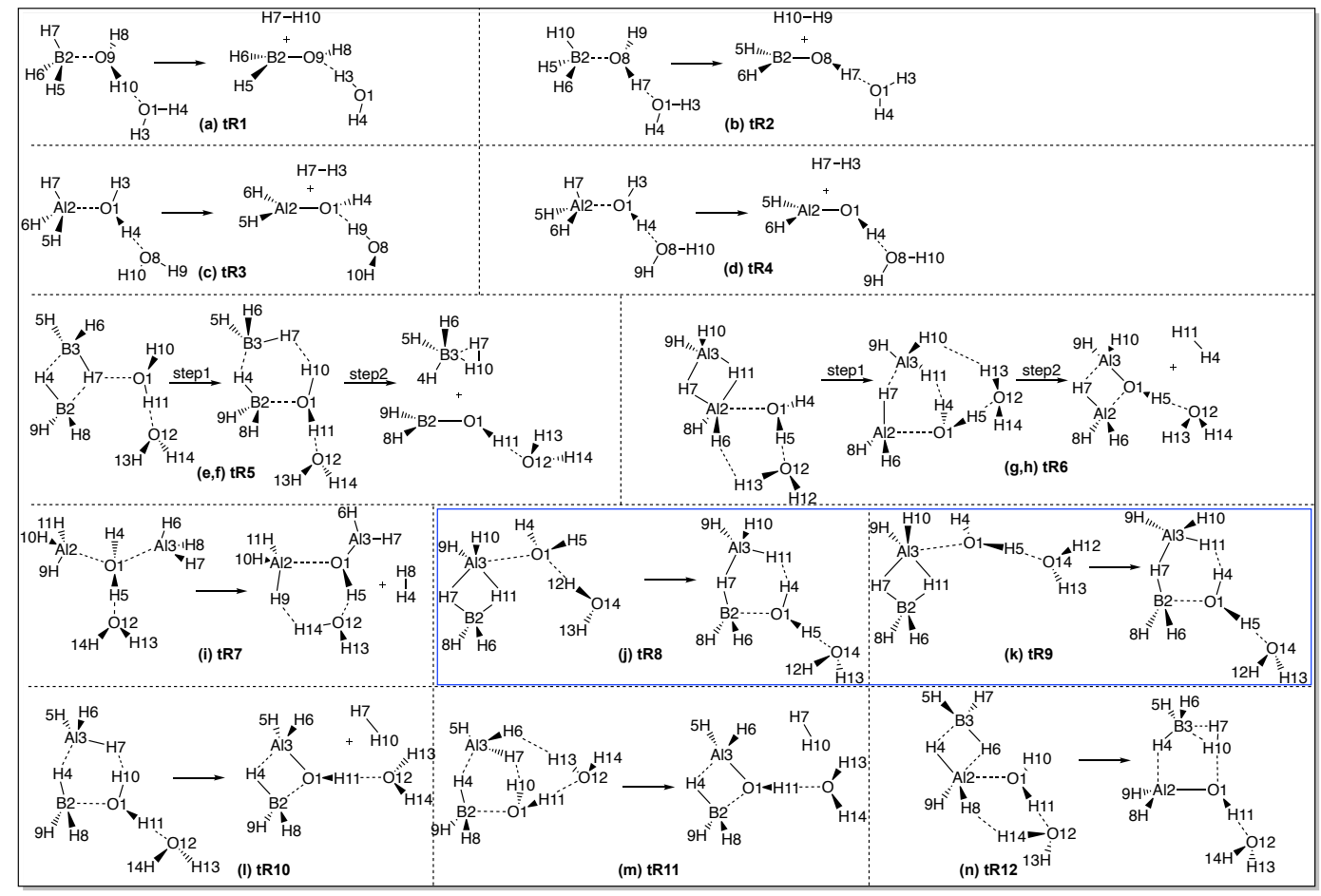

Fig. S2: Reactions tR1 - tR12 with two-water molecules investigated in this work. Reactions shown within blue boxes represent those which do not release hydrogen during the reaction. 


\section{Local mode analysis for selected bonds}

\subsection{B(Al)-H cleavage and $\mathrm{H}$-H formation for reactions $\mathrm{oR} 1-\mathrm{oR} 9$}

Table S1: Bond length, local mode force constants $\mathrm{k}^{a}, \mathrm{H}-\mathrm{H}$ and $\mathrm{B}(\mathrm{Al})-\mathrm{H}$ bond orders $B S O$, local modes frequencies $\omega^{a}$, of the reactant $\mathbf{R}$, the transition state TS, and the product $\mathbf{P}$ for reactions $\mathbf{o R 1 - o R 9}$ calculated at the MP2/6-31+G(d,p) level of theory.

\begin{tabular}{|c|c|c|c|c|c|c|}
\hline Reaction & Molecule & Bond & $\begin{array}{c}\text { Bond length } \\
\AA\end{array}$ & $\begin{array}{c}\mathrm{k}^{a} \\
\mathrm{mDyn} / \AA\end{array}$ & $B S O n$ & $\begin{array}{c}\omega^{a} \\
\mathrm{~cm}^{-1}\end{array}$ \\
\hline \multirow[t]{6}{*}{ oR1 } & \multirow[t]{2}{*}{$\mathbf{R}$} & H3-H7 & 2.556 & 0.112 & 0.114 & 614.9 \\
\hline & & B2-H7 & 1.201 & 3.688 & 0.904 & 2603.6 \\
\hline & \multirow[t]{2}{*}{ TS } & H3-H7 & 1.005 & 1.028 & 0.377 & 1861.0 \\
\hline & & B2-H7 & 1.322 & 1.932 & 0.507 & 1884.7 \\
\hline & \multirow[t]{2}{*}{$\mathbf{P}$} & $\mathrm{H} 3-\mathrm{H} 7$ & 0.735 & 6.239 & 0.994 & 4584.0 \\
\hline & & B2-H7 & 4.191 & 0.005 & 0.002 & 95.1 \\
\hline \multirow[t]{6}{*}{ oR2 } & \multirow[t]{2}{*}{$\mathbf{R}$} & H3-H7 & 2.677 & 0.061 & 0.082 & 451.6 \\
\hline & & $\mathrm{A} 12-\mathrm{H} 7$ & 1.592 & 2.141 & 0.925 & 1934.1 \\
\hline & \multirow[t]{2}{*}{ TS } & H3-H7 & 1.051 & 0.648 & 0.294 & 1477.4 \\
\hline & & Al2-H7 & 1.755 & 1.170 & 0.512 & 1429.6 \\
\hline & \multirow[t]{2}{*}{$\mathbf{P}$} & H3-H7 & 0.735 & 6.216 & 0.992 & 4575.6 \\
\hline & & $\mathrm{Al} 2-\mathrm{H} 7$ & 3.212 & 0.008 & 0.004 & 117.4 \\
\hline \multirow[t]{9}{*}{ oR3 } & \multirow[t]{3}{*}{$\mathbf{R}$} & H4-H11 & 3.030 & 0.016 & 0.040 & 230.5 \\
\hline & & B3-H11 & 1.301 & 2.071 & 0.540 & 1951.0 \\
\hline & & B2-H11 & 1.305 & 2.060 & 0.537 & 1945.8 \\
\hline & \multirow[t]{3}{*}{ TS } & H4-H11 & 2.055 & 0.074 & 0.091 & 500.8 \\
\hline & & B3-H11 & 1.206 & 3.524 & 0.868 & 2545.2 \\
\hline & & B2-H11 & 1.782 & 0.200 & 0.067 & 606.8 \\
\hline & \multirow[t]{3}{*}{$\mathbf{P}$} & H4-H11 & 1.778 & 0.072 & 0.090 & 493.6 \\
\hline & & B3-H11 & 1.208 & 3.401 & 0.841 & 2500.3 \\
\hline & & B2-H11 & 2.521 & 0.187 & 0.063 & 587.0 \\
\hline \multirow[t]{6}{*}{ oR4 } & \multirow[t]{2}{*}{$\mathbf{R}$} & H7-H10 & 2.381 & 0.068 & 0.087 & 477.0 \\
\hline & & B3-H7 & 1.203 & 3.618 & 0.889 & 2578.8 \\
\hline & \multirow[t]{2}{*}{ TS } & H7-H10 & 0.915 & 1.294 & 0.426 & 2087.7 \\
\hline & & B3-H7 & 1.304 & 1.884 & 0.496 & 1861.1 \\
\hline & \multirow[t]{2}{*}{$\mathbf{P}$} & H7-H10 & 0.787 & 3.768 & 0.758 & 3562.4 \\
\hline & & B3-H7 & 1.402 & 0.800 & 0.231 & 1212.8 \\
\hline oR5, Step 1 & $\mathbf{R}$ & H4-H11 & 2.945 & 0.042 & 0.067 & 375.7 \\
\hline & & Al3-H11 & 1.732 & 1.027 & 0.450 & 1339.4 \\
\hline & & $\mathrm{Al} 2-\mathrm{H} 11$ & 1.739 & 0.936 & 0.411 & 1279.1 \\
\hline & TS & H4-H11 & 2.090 & 0.078 & 0.094 & 513.3 \\
\hline & & Al3-H11 & 1.640 & 1.683 & 0.731 & 1714.4 \\
\hline & & Al2-H11 & 2.164 & 0.089 & 0.041 & 395.4 \\
\hline & $\mathbf{P}$ & H4-H1 1 & 1.393 & 0.164 & 0.140 & 742.2 \\
\hline & & Al3-H11 & 1.639 & 1.646 & 0.715 & 1695.7 \\
\hline & & Al2-H11 & 3.268 & 0.167 & 0.076 & 540.4 \\
\hline oR5, Step 2 & $\mathbf{R}$ & H4-H11 & 1.393 & 0.164 & 0.140 & 742.3 \\
\hline & & Al3-H11 & 1.639 & 1.646 & 0.715 & 1695.7 \\
\hline & & Al2-H11 & 3.268 & 0.167 & 0.076 & 540.5 \\
\hline & TS & H4-H11 & 0.923 & 0.985 & 0.368 & 1821.0 \\
\hline & & Al3-H11 & 1.734 & 1.127 & 0.493 & 1403.1 \\
\hline & & Al2-H11 & 3.237 & 0.289 & 0.130 & 710.3 \\
\hline & $\mathbf{P}$ & H4-H11 & 0.736 & 6.209 & 0.992 & 4573.1 \\
\hline & & Al3-H11 & 4.595 & 0.005 & 0.002 & 90.7 \\
\hline & & Al2-H11 & 4.595 & 0.005 & 0.002 & 90.7 \\
\hline oR6 & $\mathbf{R}$ & H4-H8 & 2.595 & 0.032 & 0.058 & 325.8 \\
\hline & & Al3-H8 & 1.587 & 1.925 & 0.833 & 1833.8 \\
\hline & TS & H4-H8 & 1.124 & 0.354 & 0.212 & 1091.6 \\
\hline & & Al3-H8 & 1.702 & 1.395 & 0.608 & 1561.0 \\
\hline & $\mathbf{P}$ & H4-H8 & 0.745 & 5.621 & 0.940 & 4351.1 \\
\hline & & Al3-H8 & 2.178 & 0.085 & 0.039 & 385.6 \\
\hline oR7 & $\mathbf{R}$ & H4-H11 & 2.837 & 0.031 & 0.057 & 322.5 \\
\hline & & B2-H11 & 1.258 & 2.440 & 0.625 & 2117.7 \\
\hline & & Al3-H11 & 1.768 & 0.862 & 0.379 & 1227.4 \\
\hline & TS & H4-H11 & 2.075 & 0.030 & 0.056 & 319.9 \\
\hline & & B2-H11 & 2.504 & 0.056 & 0.021 & 321.1 \\
\hline & & Al3-H11 & 1.594 & 2.108 & 0.911 & 1919.2 \\
\hline & $\mathbf{P}$ & H4-H11 & 1.457 & 0.164 & 0.140 & 743.4 \\
\hline & & B2-H11 & 2.849 & 0.221 & 0.073 & 636.9 \\
\hline
\end{tabular}




\begin{tabular}{|c|c|c|c|c|c|c|}
\hline & & Al3-H11 & 1.631 & 1.709 & 0.742 & 1728.0 \\
\hline \multirow[t]{6}{*}{ oR8 } & $\mathbf{R}$ & H7-H10 & 1.457 & 0.158 & 0.137 & 729.1 \\
\hline & & $\mathrm{Al} 3-\mathrm{H} 7$ & 1.632 & 1.692 & 0.734 & 1719.2 \\
\hline & TS & H7-H10 & 0.926 & 1.021 & 0.375 & 1854.9 \\
\hline & & $\mathrm{Al3}-\mathrm{H} 7$ & 1.735 & 1.137 & 0.497 & 1409.6 \\
\hline & $\mathbf{P}$ & H7-H10 & 0.735 & 6.246 & 0.995 & 4586.6 \\
\hline & & Al3-H7 & 4.525 & 0.005 & 0.002 & 89.5 \\
\hline \multirow[t]{12}{*}{ oR9 } & $\mathbf{R}$ & H7-H10 & 4.405 & 0.026 & 0.052 & 294.7 \\
\hline & & B3-H7 & 1.193 & 3.872 & 0.944 & 2668.0 \\
\hline & & Al2-H6 & 1.768 & 0.862 & 0.379 & 1227.4 \\
\hline & & B3-H10 & 4.010 & 0.052 & 0.020 & 308.4 \\
\hline & TS & H7-H10 & 0.889 & 1.545 & 0.469 & 2281.1 \\
\hline & & B3-H7 & 1.308 & 1.849 & 0.488 & 1843.8 \\
\hline & & Al2-H6 & 2.921 & 0.082 & 0.038 & 378.5 \\
\hline & & B3-H10 & 1.571 & 0.483 & 0.147 & 941.9 \\
\hline & $\mathbf{P}$ & H7-H10 & 0.800 & 3.391 & 0.716 & 3379.4 \\
\hline & & B3-H7 & 1.373 & 1.059 & 0.296 & 1395.6 \\
\hline & & Al2-H6 & 3.126 & 0.049 & 0.023 & 291.1 \\
\hline & & B3-H10 & 1.439 & 0.813 & 0.234 & 1222.7 \\
\hline
\end{tabular}

\section{2. $B(A l)-H$ cleavage and $H-H$ formation for reactions $t R 1-t R 12$}

Table S2: Bond length, local mode force constants $\mathrm{k}^{a}, \mathrm{H}-\mathrm{H}$ and $\mathrm{B}(\mathrm{Al})-\mathrm{H}$ bond orders $B S O$, local modes frequencies $\omega^{a}$, of the reactant $\mathbf{R}$, the transition state TS, and the product $\mathbf{P}$ for reactions tR1-tR12 calculated at the MP2/6-31+G(d,p) level of theory.

\begin{tabular}{|c|c|c|c|c|c|c|}
\hline Reaction & Molecule & Bond & $\begin{array}{c}\text { Bond length } \\
\AA\end{array}$ & $\begin{array}{c}\mathrm{k}^{a} \\
\mathrm{mDyn} / \AA\end{array}$ & $B S O n$ & $\begin{array}{c}\omega^{a} \\
\mathrm{~cm}^{-1}\end{array}$ \\
\hline \multirow[t]{6}{*}{ tR1 } & \multirow[t]{2}{*}{$\mathbf{R}$} & H7-H10 & 2.436 & 0.177 & 0.146 & 771.4 \\
\hline & & B2-H7 & 1.209 & 3.444 & 0.850 & 2516.3 \\
\hline & \multirow[t]{2}{*}{ TS } & H7-H10 & 1.017 & 0.963 & 0.364 & 1801.2 \\
\hline & & B2-H7 & 1.311 & 2.052 & 0.535 & 1942.1 \\
\hline & \multirow[t]{2}{*}{$\mathbf{P}$} & H7-H10 & 0.735 & 6.255 & 0.995 & 4590.0 \\
\hline & & B2-H7 & 4.183 & 0.006 & 0.003 & 101.9 \\
\hline \multirow[t]{6}{*}{ tR2 } & \multirow[t]{2}{*}{$\mathbf{R}$} & H9-H10 & 2.569 & 0.136 & 0.127 & 677.7 \\
\hline & & B2-H10 & 1.209 & 3.444 & 0.850 & 2516.3 \\
\hline & \multirow[t]{2}{*}{ TS } & H9-H10 & 0.991 & 1.094 & 0.390 & 1919.9 \\
\hline & & B2-H10 & 1.335 & 1.785 & 0.473 & 1811.6 \\
\hline & \multirow[t]{2}{*}{$\mathbf{P}$} & H9-H10 & 0.736 & 6.214 & 0.992 & 4575.0 \\
\hline & & B2-H10 & 4.201 & 0.004 & 0.002 & 90.0 \\
\hline \multirow[t]{6}{*}{ tR3 } & \multirow[t]{2}{*}{$\mathbf{R}$} & H3-H7 & 2.761 & 0.072 & 0.090 & 491.1 \\
\hline & & Al2-H7 & 1.590 & 2.151 & 0.929 & 1938.7 \\
\hline & \multirow[t]{2}{*}{ TS } & H3-H7 & 1.065 & 0.591 & 0.280 & 1410.4 \\
\hline & & Al2-H7 & 1.743 & 1.213 & 0.530 & 1455.5 \\
\hline & \multirow[t]{2}{*}{$\mathbf{P}$} & H3-H7 & 0.736 & 6.204 & 0.991 & 4571.3 \\
\hline & & $\mathrm{Al} 2-\mathrm{H} 7$ & 3.168 & 0.010 & 0.005 & 130.3 \\
\hline \multirow[t]{6}{*}{ tR4 } & \multirow[t]{2}{*}{$\mathbf{R}$} & H3-H7 & 2.761 & 0.072 & 0.090 & 491.1 \\
\hline & & A12-H7 & 1.590 & 2.151 & 0.929 & 1938.7 \\
\hline & \multirow[t]{2}{*}{ TS } & H3-H7 & 1.028 & 0.721 & 0.311 & 1558.2 \\
\hline & & Al2-H7 & 1.772 & 1.092 & 0.478 & 1381.4 \\
\hline & \multirow[t]{2}{*}{$\mathbf{P}$} & H3-H7 & 0.735 & 6.216 & 0.992 & 4575.8 \\
\hline & & Al2-H7 & 3.285 & 0.007 & 0.003 & 110.9 \\
\hline \multirow[t]{9}{*}{ tR5, Step 1} & \multirow[t]{3}{*}{$\mathbf{R}$} & H7-H10 & 3.097 & 0.040 & 0.066 & 365.9 \\
\hline & & B2-H7 & 1.302 & 2.116 & 0.550 & 1972.1 \\
\hline & & B3-H7 & 1.307 & 2.023 & 0.529 & 1928.4 \\
\hline & \multirow[t]{3}{*}{ TS } & H7-H10 & 2.138 & 0.060 & 0.082 & 449.9 \\
\hline & & B2-H7 & 1.710 & 0.211 & 0.070 & 623.0 \\
\hline & & B3-H7 & 1.208 & 3.487 & 0.860 & 2531.8 \\
\hline & \multirow[t]{3}{*}{$\mathbf{P}$} & H7-H10 & 1.880 & 0.059 & 0.081 & 445.6 \\
\hline & & B2-H7 & 2.510 & 0.135 & 0.047 & 499.1 \\
\hline & & B3-H7 & 1.206 & 3.506 & 0.864 & 2538.6 \\
\hline \multirow[t]{6}{*}{ tR5, Step 2} & \multirow[t]{3}{*}{$\mathbf{R}$} & H7-H10 & 1.939 & 0.067 & 0.087 & 474.7 \\
\hline & & B2-H7 & 2.527 & 0.197 & 0.066 & 601.1 \\
\hline & & B3-H7 & 1.204 & 3.536 & 0.871 & 2549.4 \\
\hline & \multirow[t]{3}{*}{ TS } & H7-H10 & 0.904 & 1.269 & 0.422 & 2067.4 \\
\hline & & B2-H7 & 2.668 & 0.402 & 0.125 & 859.3 \\
\hline & & B3-H7 & 1.310 & 1.799 & 0.476 & 1818.4 \\
\hline
\end{tabular}




\begin{tabular}{|c|c|c|c|c|c|c|}
\hline & $\mathbf{P}$ & $\begin{array}{c}\text { H7-H10 } \\
\text { B2-H7 } \\
\text { B3-H7 }\end{array}$ & $\begin{array}{l}0.792 \\
3.873 \\
1.385\end{array}$ & $\begin{array}{l}3.748 \\
0.042 \\
0.964\end{array}$ & $\begin{array}{l}0.756 \\
0.017 \\
0.272\end{array}$ & $\begin{array}{r}3553.0 \\
279.3 \\
1331.4\end{array}$ \\
\hline \multirow[t]{9}{*}{ tR6, Step 1} & \multirow[t]{3}{*}{$\mathbf{R}$} & H4-H11 & 3.285 & 0.096 & 0.105 & 568.4 \\
\hline & & Al2-H11 & 1.724 & 0.992 & 0.435 & 1316.7 \\
\hline & & Al3-H11 & 1.729 & 1.031 & 0.452 & 1341.9 \\
\hline & \multirow[t]{3}{*}{ TS } & H4-H11 & 2.293 & 0.117 & 0.117 & 628.2 \\
\hline & & Al2-H11 & 2.119 & 0.072 & 0.033 & 355.1 \\
\hline & & Al3-H11 & 1.639 & 1.618 & 0.703 & 1681.1 \\
\hline & \multirow[t]{3}{*}{$\mathbf{P}$} & H4-H11 & 1.801 & 0.040 & 0.066 & 367.4 \\
\hline & & Al2-H11 & 3.261 & 0.114 & 0.052 & 446.0 \\
\hline & & $\mathrm{Al} 3-\mathrm{H} 11$ & 1.614 & 1.831 & 0.794 & 1788.4 \\
\hline \multirow[t]{9}{*}{ tR6, Step 2} & \multirow[t]{3}{*}{$\mathbf{R}$} & H4-H11 & 1.823 & 0.034 & 0.060 & 340.8 \\
\hline & & Al2-H11 & 3.257 & 0.109 & 0.050 & 436.6 \\
\hline & & Al3-H11 & 1.614 & 1.828 & 0.792 & 1787.0 \\
\hline & \multirow[t]{3}{*}{ TS } & H4-H11 & 0.873 & 1.498 & 0.461 & 2245.9 \\
\hline & & Al2-H11 & 3.265 & 0.266 & 0.120 & 681.3 \\
\hline & & Al3-H11 & 1.765 & 0.997 & 0.437 & 1320.1 \\
\hline & \multirow[t]{3}{*}{$\mathbf{P}$} & H4-H11 & 0.735 & 6.242 & 0.994 & 4585.1 \\
\hline & & $\mathrm{Al} 2-\mathrm{H} 11$ & 5.326 & 0.000 & 0.000 & 28.1 \\
\hline & & Al3-H11 & 4.260 & 0.001 & 0.001 & 35.9 \\
\hline \multirow[t]{6}{*}{ tR7 } & \multirow[t]{2}{*}{$\mathbf{R}$} & H4-H8 & 2.678 & 0.049 & 0.073 & 406.7 \\
\hline & & Al3-H8 & 1.591 & 2.140 & 0.925 & 1933.7 \\
\hline & \multirow[t]{2}{*}{ TS } & H4-H8 & 1.110 & 0.435 & 0.237 & 1210.0 \\
\hline & & Al3-H8 & 1.713 & 1.358 & 0.592 & 1540.5 \\
\hline & \multirow[t]{2}{*}{$\mathbf{P}$} & H4-H8 & 0.738 & 5.853 & 0.961 & 4440.1 \\
\hline & & Al3-H8 & 2.676 & 0.005 & 0.002 & 96.0 \\
\hline \multirow[t]{9}{*}{ tR8 } & \multirow[t]{3}{*}{$\mathbf{R}$} & H4-H11 & 3.315 & 0.032 & 0.058 & 327.3 \\
\hline & & Al3-H11 & 1.836 & 0.397 & 0.177 & 832.3 \\
\hline & & B2-H11 & 1.236 & 2.710 & 0.686 & 2232.0 \\
\hline & \multirow{3}{*}{ TS } & H4-H11 & 1.881 & 0.044 & 0.069 & 384.0 \\
\hline & & Al3-H11 & 1.596 & 2.084 & 0.901 & 1908.2 \\
\hline & & B2-H11 & 2.593 & 0.055 & 0.021 & 318.3 \\
\hline & $\mathbf{P}$ & H4-H11 & 1.610 & 0.093 & 0.103 & 558.9 \\
\hline & & Al3-H11 & 1.621 & 1.757 & 0.762 & 1752.0 \\
\hline & & B2-H11 & 2.859 & 0.230 & 0.076 & 650.1 \\
\hline tR9 & $\mathbf{R}$ & H4-H11 & 3.057 & 0.055 & 0.078 & 429.7 \\
\hline & & Al3-H11 & 1.779 & 0.726 & 0.320 & 1126.0 \\
\hline & & B2-H11 & 1.254 & 2.432 & 0.623 & 2114.6 \\
\hline & TS & H4-H11 & 2.184 & 0.030 & 0.056 & 317.7 \\
\hline & & Al3-H11 & 1.592 & 2.122 & 0.917 & 1925.4 \\
\hline & & B2-H11 & 2.412 & 0.049 & 0.019 & 299.9 \\
\hline & $\mathbf{P}$ & H4-H11 & 1.602 & 0.117 & 0.117 & 626.9 \\
\hline & & Al3-H11 & 1.622 & 1.803 & 0.782 & 1775.0 \\
\hline & & B2-H11 & 2.848 & 0.225 & 0.074 & 642.5 \\
\hline tR10 & $\mathbf{R}$ & H7-H10 & 1.594 & 0.121 & 0.119 & 638.6 \\
\hline & & Al3-H7 & 1.622 & 1.803 & 0.782 & 1774.5 \\
\hline & TS & H7-H10 & 0.964 & 0.642 & 0.292 & 1470.3 \\
\hline & & Al3-H7 & 1.716 & 1.149 & 0.503 & 1416.6 \\
\hline & $\mathbf{P}$ & H7-H10 & 0.735 & 6.245 & 0.995 & 4586.4 \\
\hline & & Al3-H7 & 3.682 & 0.000 & 0.000 & 28.8 \\
\hline tR11 & $\mathbf{R}$ & H7-H10 & 1.840 & 0.047 & 0.072 & 398.4 \\
\hline & & Al3-H7 & 1.605 & 1.926 & 0.834 & 1834.4 \\
\hline & TS & H7-H10 & 0.877 & 1.523 & 0.404 & 2265.1 \\
\hline & & Al3-H7 & 1.766 & 0.990 & 0.434 & 1314.9 \\
\hline & $\mathbf{P}$ & H7-H10 & 0.735 & 6.236 & 0.994 & 4582.9 \\
\hline & & Al3-H7 & 4.174 & 0.008 & 0.004 & 119.8 \\
\hline tR12 & $\mathbf{R}$ & H7-H10 & 4.375 & 0.040 & 0.066 & 368.5 \\
\hline & & B3-H7 & 1.193 & 3.870 & 0.944 & 2667.2 \\
\hline & TS & H7-H10 & 0.847 & 2.024 & 0.542 & 2610.8 \\
\hline & & B3-H7 & 1.329 & 1.568 & 0.421 & 1697.7 \\
\hline & $\mathbf{P}$ & H7-H10 & 0.811 & 2.477 & 0.605 & 2888.6 \\
\hline & & B3-H7 & 1.359 & 1.069 & 0.299 & 1401.5 \\
\hline
\end{tabular}




\section{3. $O-B(A l)$ formation for reactions oR1-oR9}

Table S3: Bond length, local mode force constants $\mathrm{k}^{a}$, O-B(Al) bond orders $B S O n$, local modes frequencies $\omega^{a}$, of the reactant $\mathbf{R}$, the transition state TS, and the product $\mathbf{P}$, for $\mathrm{O}-\mathrm{B}(\mathrm{Al})$ formation in reactions $\mathbf{o R} 1-\mathbf{o R 9}$ calculated at the MP2/6-31+G(d,p) level of theory.

\begin{tabular}{|c|c|c|c|c|c|c|}
\hline Reaction & Molecule & Bond & $\begin{array}{c}\text { Bond length } \\
\AA\end{array}$ & $\begin{array}{c}\mathrm{k}^{a} \\
\mathrm{mDyn} / \AA\end{array}$ & $B S O n$ & $\begin{array}{c}\omega^{a} \\
\mathrm{~cm}^{-1}\end{array}$ \\
\hline \multirow[t]{3}{*}{ oR1 } & $\mathbf{R}$ & O1-B2 & 1.743 & 0.769 & 0.253 & 447.3 \\
\hline & TS & O1-B2 & 1.606 & 2.707 & 0.610 & 839.3 \\
\hline & $\mathbf{P}$ & O1-B2 & 1.365 & 6.639 & 1.143 & 1314.6 \\
\hline \multirow[t]{3}{*}{ oR2 } & $\mathbf{R}$ & $\mathrm{O} 1-\mathrm{Al} 2$ & 2.080 & 0.777 & 0.513 & 362.3 \\
\hline & TS & $\mathrm{O} 1-\mathrm{A} 12$ & 1.917 & 2.049 & 0.881 & 588.5 \\
\hline & $\mathbf{P}$ & $\mathrm{O} 1-\mathrm{Al} 2$ & 1.730 & 3.773 & 1.238 & 798.6 \\
\hline \multirow[t]{3}{*}{ oR3 } & $\mathbf{R}$ & O1-B2 & 3.169 & 0.009 & 0.011 & 48.0 \\
\hline & TS & O1-B2 & 1.889 & 0.395 & 1.159 & 320.6 \\
\hline & $\mathbf{P}$ & O1-B2 & 1.603 & 1.801 & 0.459 & 684.7 \\
\hline \multirow[t]{3}{*}{ oR4 } & $\mathbf{R}$ & O1-B2 & 1.603 & 1.801 & 0.459 & 684.7 \\
\hline & TS & O1-B2 & 1.487 & 3.829 & 0.778 & 998.3 \\
\hline & $\mathbf{P}$ & O1-B2 & 1.368 & 6.501 & 1.126 & 1300.8 \\
\hline \multirow[t]{3}{*}{ oR5, Step 1} & $\mathbf{R}$ & $\mathrm{O} 1-\mathrm{Al} 2$ & 2.155 & 0.442 & 0.374 & 273.2 \\
\hline & TS & $\mathrm{O} 1-\mathrm{Al} 2$ & 2.028 & 0.881 & 0.550 & 386.0 \\
\hline & $\mathbf{P}$ & $\mathrm{O} 1-\mathrm{A} 12$ & 1.957 & 1.284 & 0.679 & 465.9 \\
\hline \multirow[t]{6}{*}{ oR5, Step 2} & $\mathbf{R}$ & $\mathrm{O} 1-\mathrm{A} 12$ & 1.957 & 1.284 & 0.679 & 465.9 \\
\hline & & $\mathrm{O} 1-\mathrm{Al} 3$ & 3.218 & 0.071 & 0.135 & 109.7 \\
\hline & TS & $\mathrm{O} 1-\mathrm{Al} 2$ & 1.854 & 2.440 & 0.971 & 642.2 \\
\hline & & $\mathrm{O} 1-\mathrm{Al} 3$ & 3.042 & 0.195 & 0.237 & 181.7 \\
\hline & $\mathbf{P}$ & $\mathrm{O} 1-\mathrm{Al} 2$ & 1.886 & 1.719 & 0.798 & 539.1 \\
\hline & & $\mathrm{O} 1-\mathrm{A} 13$ & 1.886 & 1.719 & 0.798 & 539.1 \\
\hline \multirow[t]{6}{*}{ oR6 } & $\mathbf{R}$ & $\mathrm{O} 1-\mathrm{Al} 3$ & 2.232 & 0.345 & 0.326 & 241.5 \\
\hline & & $\mathrm{O} 1-\mathrm{Al} 2$ & 2.214 & 0.359 & 0.333 & 246.2 \\
\hline & TS & $\mathrm{O} 1-\mathrm{Al} 3$ & 2.075 & 1.012 & 0.594 & 413.6 \\
\hline & & $\mathrm{O} 1-\mathrm{Al} 2$ & 2.097 & 0.688 & 0.479 & 341.0 \\
\hline & $\mathbf{P}$ & $\mathrm{O} 1-\mathrm{Al} 3$ & 1.793 & 2.828 & 1.054 & 691.4 \\
\hline & & $\mathrm{O} 1-\mathrm{A} 12$ & 2.003 & 1.036 & 0.602 & 418.5 \\
\hline \multirow[t]{3}{*}{ oR7 } & $\mathbf{R}$ & O1-B2 & 3.647 & 0.259 & 0.118 & 259.9 \\
\hline & TS & O1-B2 & 2.777 & 0.078 & 0.051 & 142.5 \\
\hline & $\mathbf{P}$ & O1-B2 & 1.620 & 1.647 & 0.431 & 654.7 \\
\hline \multirow[t]{6}{*}{ oR8 } & $\mathbf{R}$ & O1-B2 & 1.609 & 1.700 & 0.441 & 665.1 \\
\hline & & $\mathrm{O} 1-\mathrm{Al} 3$ & 3.130 & 0.375 & 0.342 & 251.6 \\
\hline & TS & O1-B2 & 1.521 & 2.930 & 0.645 & 873.4 \\
\hline & & $\mathrm{O} 1-\mathrm{A} 13$ & 2.823 & 0.265 & 0.281 & 211.6 \\
\hline & $\mathbf{P}$ & O1-B2 & 1.583 & 1.987 & 0.492 & 719.1 \\
\hline & & $\mathrm{O} 1-\mathrm{Al} 3$ & 1.868 & 1.920 & 0.849 & 569.6 \\
\hline \multirow[t]{3}{*}{ oR9 } & $\mathbf{R}$ & $\mathrm{O} 1-\mathrm{Al} 2$ & 2.109 & 0.594 & 0.441 & 316.8 \\
\hline & TS & $\mathrm{O} 1-\mathrm{Al} 2$ & 1.823 & 2.860 & 1.061 & 695.2 \\
\hline & $\mathbf{P}$ & $\mathrm{O} 1-\mathrm{Al} 2$ & 1.772 & 2.874 & 1.063 & 697.0 \\
\hline
\end{tabular}




\subsection{O-B(Al) formation for reactions $t R 1-t R 12$}

Table S4: Bond length, local mode force constants $\mathrm{k}^{a}, \mathrm{O}-\mathrm{B}(\mathrm{Al})$ bond orders $B S O n$, local modes frequencies $\omega^{a}$, of the reactant $\mathbf{R}$, the transition state TS, and the product $\mathbf{P}$, for O-B(Al) formation in reactions tR1-tR12 calculated at the MP2/6-31+G(d,p) level of theory.

\begin{tabular}{|c|c|c|c|c|c|c|}
\hline Reaction & Molecule & Bond & $\begin{array}{c}\text { Bond length } \\
\AA\end{array}$ & $\begin{array}{c}\mathrm{k}^{a} \\
\mathrm{mDyn} / \AA\end{array}$ & $B S O n$ & $\begin{array}{c}\omega^{a} \\
\mathrm{~cm}^{-1}\end{array}$ \\
\hline \multirow[t]{3}{*}{ tR1 } & $\mathbf{R}$ & O9-B2 & 1.680 & 1.181 & 0.342 & 554.5 \\
\hline & TS & O9-B2 & 1.626 & 2.445 & 0.568 & 797.8 \\
\hline & $\mathbf{P}$ & O9-B2 & 1.371 & 6.371 & 1.110 & 1287.7 \\
\hline \multirow[t]{3}{*}{ tR2 } & $\mathbf{R}$ & O8-B2 & 1.680 & 1.181 & 0.342 & 554.5 \\
\hline & TS & O8-B2 & 1.577 & 2.951 & 0.648 & 876.4 \\
\hline & $\mathbf{P}$ & O8-B2 & 1.357 & 6.898 & 1.174 & 1340.0 \\
\hline \multirow[t]{3}{*}{ tR3 } & $\mathbf{R}$ & $\mathrm{O} 1-\mathrm{A} 12$ & 2.019 & 1.022 & 0.597 & 415.7 \\
\hline & TS & $\mathrm{O} 1-\mathrm{A} 12$ & 1.943 & 1.725 & 0.800 & 540.0 \\
\hline & $\mathbf{P}$ & $\mathrm{O} 1-\mathrm{Al} 2$ & 1.745 & 3.529 & 1.192 & 772.3 \\
\hline \multirow[t]{3}{*}{ tR4 } & $\mathbf{R}$ & $\mathrm{O} 1-\mathrm{A} 12$ & 2.019 & 1.022 & 0.597 & 415.7 \\
\hline & TS & $\mathrm{O} 1-\mathrm{Al} 2$ & 1.879 & 2.312 & 0.942 & 625.2 \\
\hline & $\mathbf{P}$ & $\mathrm{O} 1-\mathrm{A} 12$ & 1.720 & 3.831 & 1.248 & 804.7 \\
\hline \multirow[t]{3}{*}{ tR5, Step 1} & $\mathbf{R}$ & O1-B2 & 3.199 & 0.034 & 0.029 & 94.2 \\
\hline & TS & O1-B2 & 1.790 & 0.575 & 0.206 & 386.9 \\
\hline & $\mathbf{P}$ & O1-B2 & 1.570 & 1.964 & 0.488 & 715.1 \\
\hline \multirow[t]{3}{*}{ tR5, Step 2} & $\mathbf{R}$ & O1-B2 & 1.568 & 2.210 & 0.529 & 758.5 \\
\hline & TS & O1-B2 & 1.455 & 4.251 & 0.837 & 1051.9 \\
\hline & $\mathbf{P}$ & O1-B2 & 1.361 & 6.706 & 1.151 & 1321.2 \\
\hline \multirow[t]{6}{*}{ tR6, Step 1} & $\mathbf{R}$ & $\mathrm{O} 1-\mathrm{A} 12$ & 2.073 & 0.690 & 0.480 & 341.4 \\
\hline & & O1-Al3 & 4.191 & 0.234 & 0.263 & 198.9 \\
\hline & TS & $\mathrm{O} 1-\mathrm{Al} 2$ & 1.962 & 1.130 & 0.632 & 437.0 \\
\hline & & $\mathrm{O} 1-\mathrm{Al} 3$ & 3.531 & 0.224 & 0.256 & 194.7 \\
\hline & $\mathbf{P}$ & $\mathrm{O} 1-\mathrm{Al} 2$ & 1.931 & 1.534 & 0.749 & 509.2 \\
\hline & & $\mathrm{O} 1-\mathrm{Al} 3$ & 3.192 & 0.149 & 0.204 & 158.7 \\
\hline \multirow[t]{6}{*}{ tR6, Step 2} & $\mathbf{R}$ & $\mathrm{O} 1-\mathrm{A} 12$ & 1.931 & 1.530 & 0.748 & 508.6 \\
\hline & & $\mathrm{O} 1-\mathrm{A} 13$ & 3.205 & 0.133 & 0.192 & 150.2 \\
\hline & TS & $\mathrm{O} 1-\mathrm{A} 12$ & 1.818 & 2.683 & 1.023 & 673.4 \\
\hline & & $\mathrm{O} 1-\mathrm{Al} 3$ & 2.967 & 0.145 & 0.201 & 156.4 \\
\hline & $\mathbf{P}$ & $\mathrm{O} 1-\mathrm{A} 12$ & 1.872 & 1.870 & 0.837 & 562.2 \\
\hline & & O1-A13 & 1.872 & 1.855 & 0.833 & 560.0 \\
\hline \multirow[t]{6}{*}{ tR7 } & $\mathbf{R}$ & $\mathrm{O} 1-\mathrm{Al} 2$ & 2.127 & 0.570 & 0.431 & 310.3 \\
\hline & & $\mathrm{O} 1-\mathrm{Al} 3$ & 2.138 & 0.546 & 0.421 & 303.9 \\
\hline & TS & $\mathrm{O} 1-\mathrm{Al} 2$ & 2.039 & 0.910 & 0.560 & 392.2 \\
\hline & & $\mathrm{O} 1-\mathrm{Al} 3$ & 2.018 & 1.228 & 0.662 & 455.5 \\
\hline & $\mathbf{P}$ & $\mathrm{O} 1-\mathrm{Al} 2$ & 1.977 & 1.161 & 0.641 & 442.9 \\
\hline & & $\mathrm{O} 1-\mathrm{Al} 3$ & 1.770 & 3.091 & 1.107 & 722.8 \\
\hline \multirow[t]{3}{*}{ tR8 } & $\mathbf{R}$ & O1-B2 & 3.339 & 0.120 & 0.069 & 176.8 \\
\hline & TS & O1-B2 & 2.890 & 0.059 & 0.042 & 124.3 \\
\hline & $\mathbf{P}$ & O1-B2 & 1.584 & 2.021 & 0.497 & 725.2 \\
\hline \multirow[t]{3}{*}{ tR9 } & $\mathbf{R}$ & O1-B2 & 3.593 & 0.145 & 0.079 & 194.6 \\
\hline & TS & O1-B2 & 2.686 & 0.077 & 0.051 & 141.4 \\
\hline & $\mathbf{P}$ & O1-B2 & 1.593 & 1.970 & 0.489 & 716.0 \\
\hline \multirow[t]{6}{*}{ tR10 } & $\mathbf{R}$ & O1-B2 & 1.594 & 1.969 & 0.488 & 715.9 \\
\hline & & $\mathrm{O} 1-\mathrm{Al} 3$ & 3.119 & 0.321 & 0.313 & 233.0 \\
\hline & TS & O1-B2 & 1.545 & 2.448 & 0.569 & 798.2 \\
\hline & & $\mathrm{O} 1-\mathrm{Al} 3$ & 2.862 & 0.266 & 0.282 & 212.2 \\
\hline & $\mathbf{P}$ & O1-B2 & 1.566 & 2.184 & 0.525 & 754.0 \\
\hline & & $\mathrm{O} 1-\mathrm{Al} 3$ & 1.844 & 2.117 & 0.897 & 598.1 \\
\hline \multirow[t]{6}{*}{ tR11 } & $\mathbf{R}$ & $\mathrm{O} 1-\mathrm{Al} 3$ & 3.072 & 0.184 & 0.230 & 176.3 \\
\hline & & O1-B2 & 1.581 & 2.026 & 0.498 & 726.2 \\
\hline & TS & $\mathrm{O} 1-\mathrm{A} 13$ & 2.753 & 0.258 & 0.277 & 208.7 \\
\hline & & O1-B2 & 1.488 & 3.074 & 0.667 & 894.5 \\
\hline & $\mathbf{P}$ & $\mathrm{O} 1-\mathrm{Al} 3$ & 1.850 & 2.106 & 0.894 & 596.7 \\
\hline & & O1-B2 & 1.567 & 2.174 & 0.523 & 752.3 \\
\hline \multirow[t]{2}{*}{ tR12 } & $\mathbf{R}$ & $\mathrm{O} 1-\mathrm{Al} 2$ & 2.034 & 0.849 & 0.539 & 378.7 \\
\hline & TS & $\mathrm{O} 1-\mathrm{A} 12$ & 1.785 & 2.989 & 1.087 & 710.8 \\
\hline
\end{tabular}




\section{Scalar curvature decomposition into selected bond components}

\subsection{Decomposition into bond components for reactions oR1-oR9}

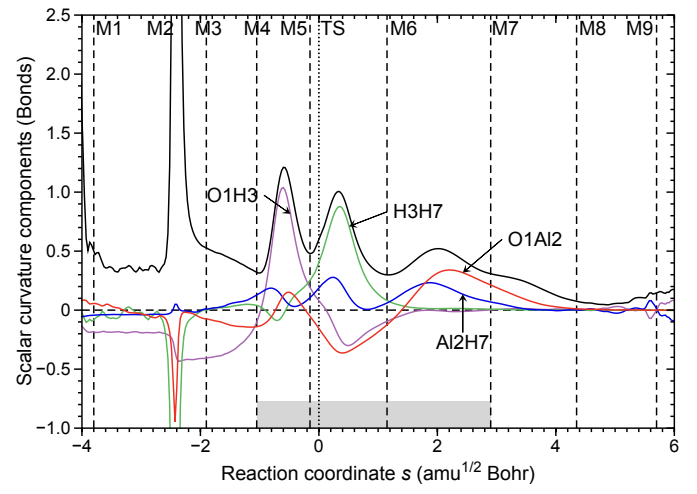

(a) Reaction oR1, PCM

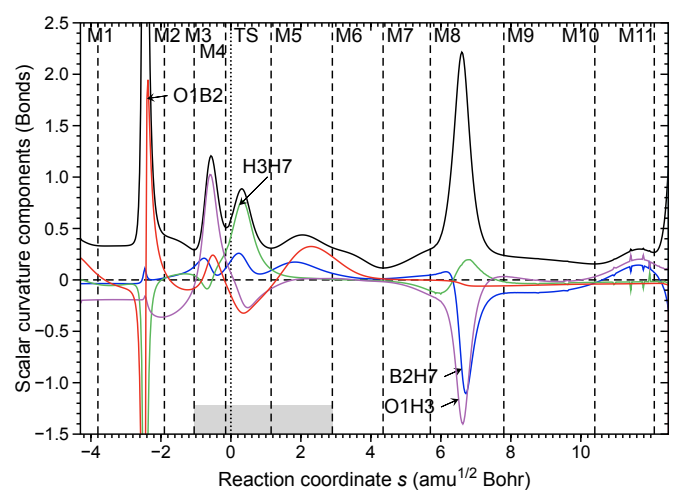

(b) Reaction $\mathbf{o R} 1$

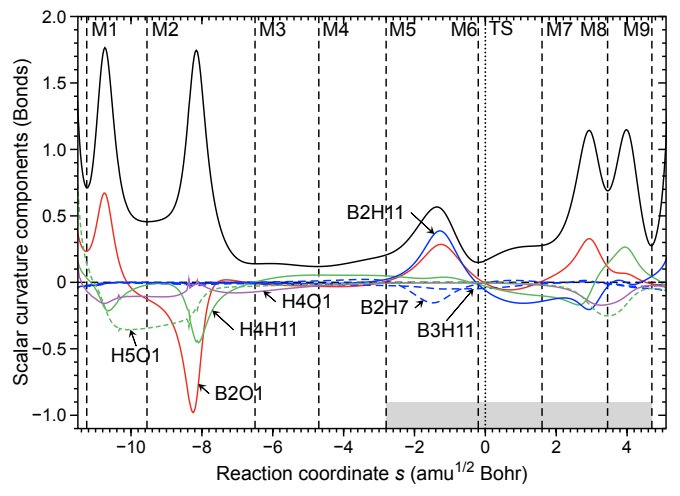

(d) Reaction $\mathbf{0 R 3}$

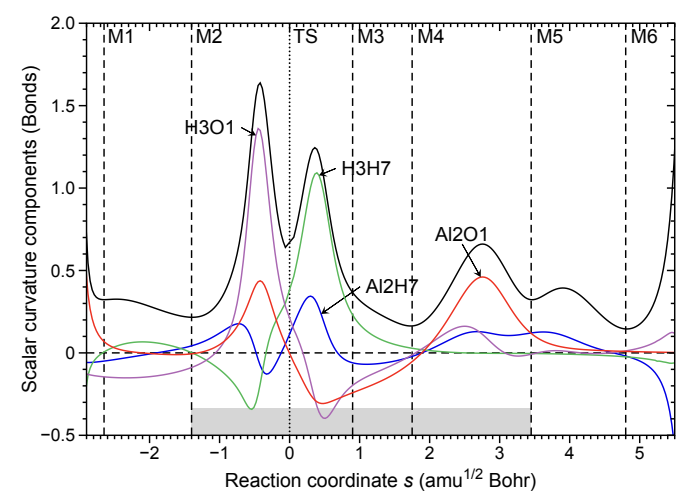

(c) Reaction $\mathbf{O R 2}$

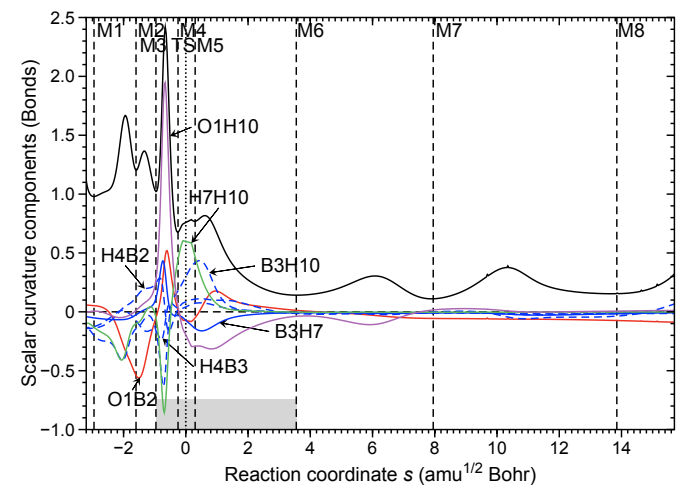

(e) Reaction $\mathbf{0 R 4}$ 


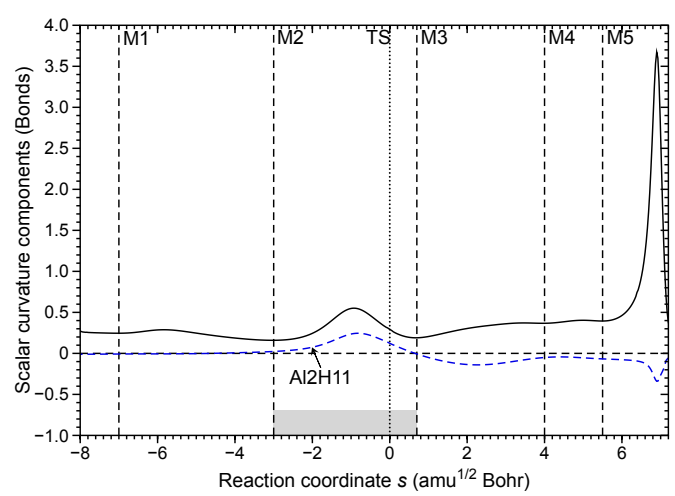

(f) Reaction oR5, Step 1

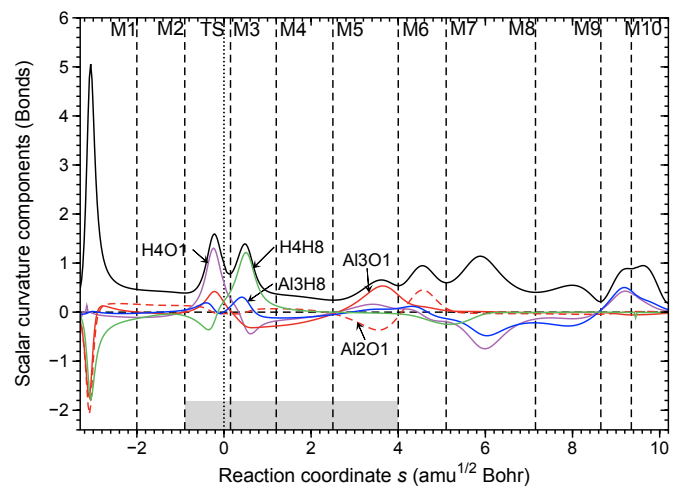

(h) Reaction oR6

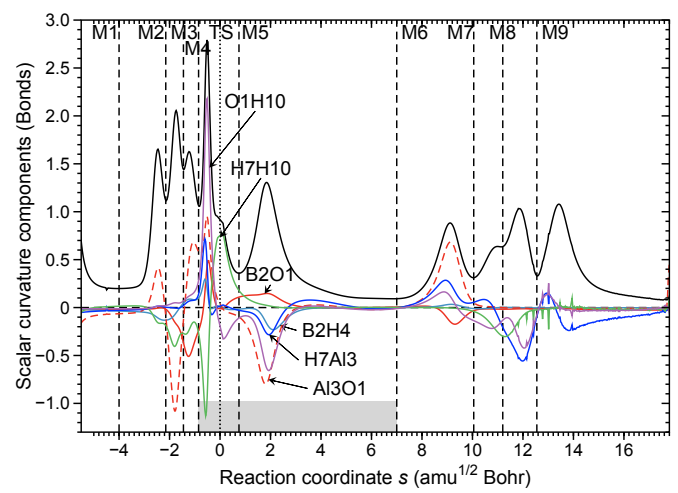

(j) Reaction $\mathbf{o R 8}$

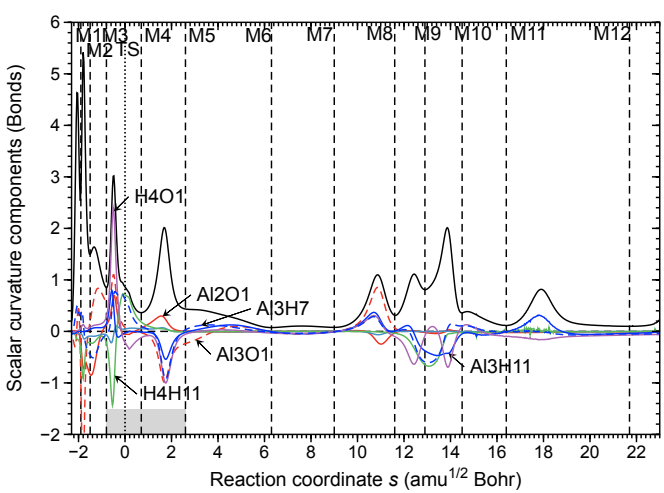

(g) Reaction oR5, Step 2

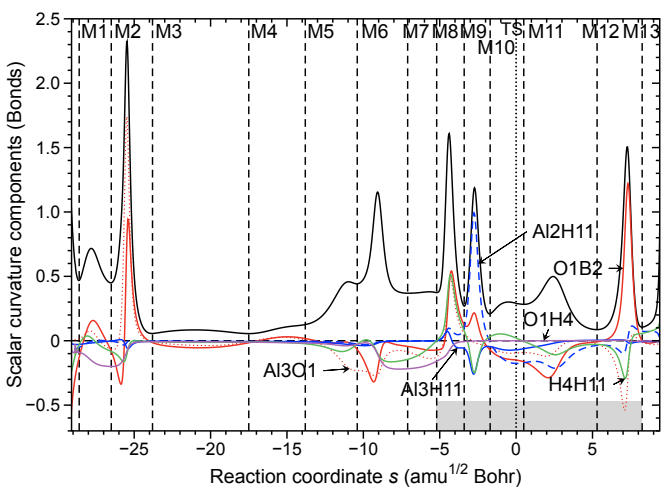

(i) Reaction oR7

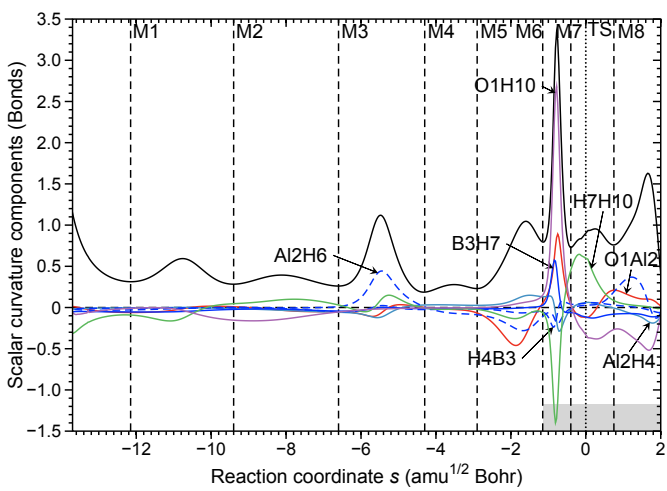

(k) Reaction $\mathbf{o R 9}$

Fig. S3: Scalar curvature as a function of the reaction path parameter $s$ (solid black line) for the hydrogen release in the presence of one-water molecule for oR1-oR9. The decomposition of the scalar curvature into the most important bond components is given. The borders of the reaction phases are indicated by vertical dashed lines at curvature minima M1, $\mathrm{M} 2, \mathrm{M} 3$, etc. The TS at $s=0 \mathrm{amu}^{1 / 2} \mathrm{~b}$ is also indicated by a vertical dotted line. The chemical phases are highlighted with a gray bar. 


\subsection{Decomposition into bond components for reactions $t R 1-t R 12$}

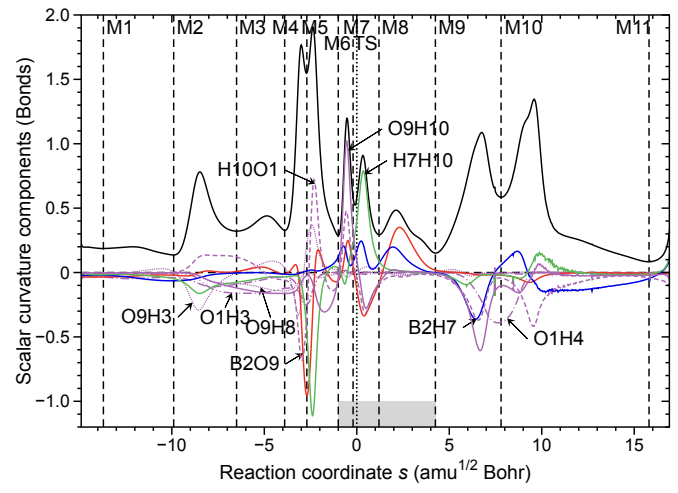

(a) Reaction tR1

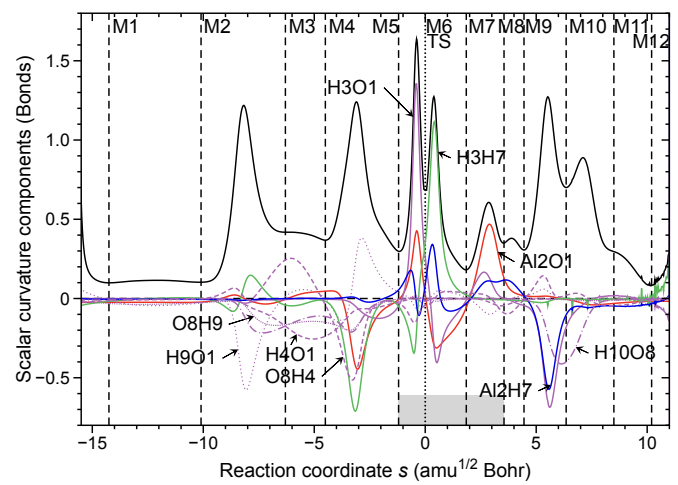

(c) Reaction tR3

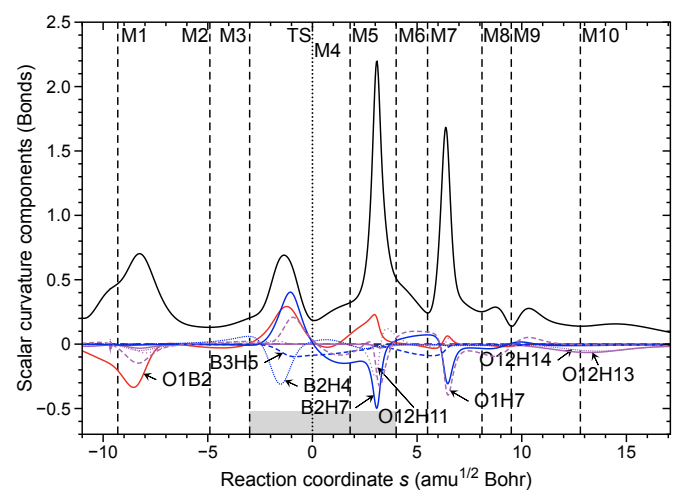

(e) Reaction tR5, Step 1

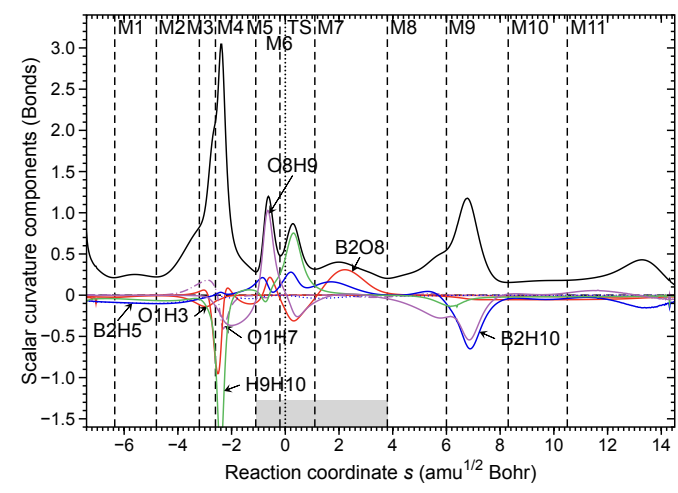

(b) Reaction tR2

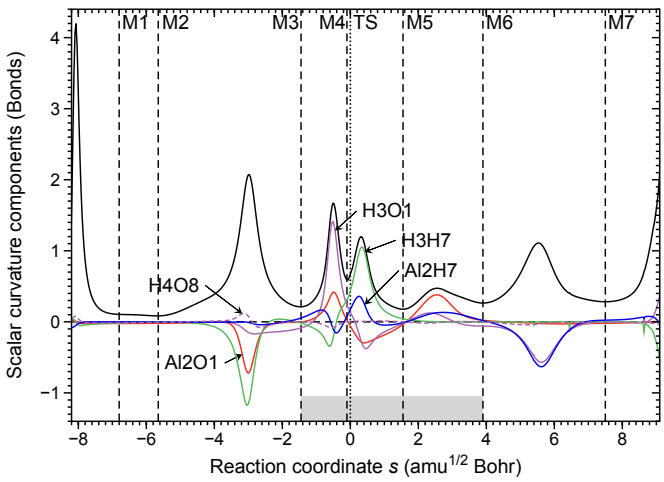

(d) Reaction tR4

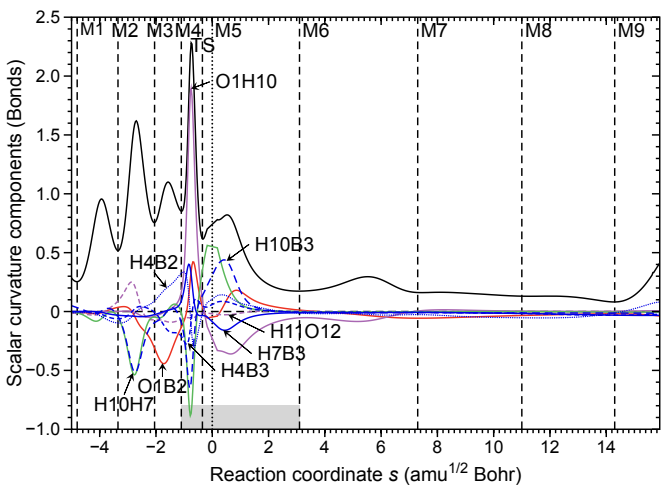

(f) Reaction tR5, Step 2 


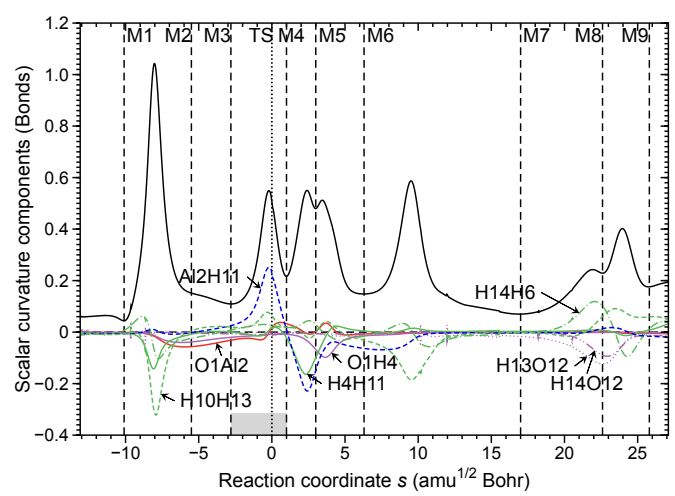

(g) Reaction tR6, Step 1

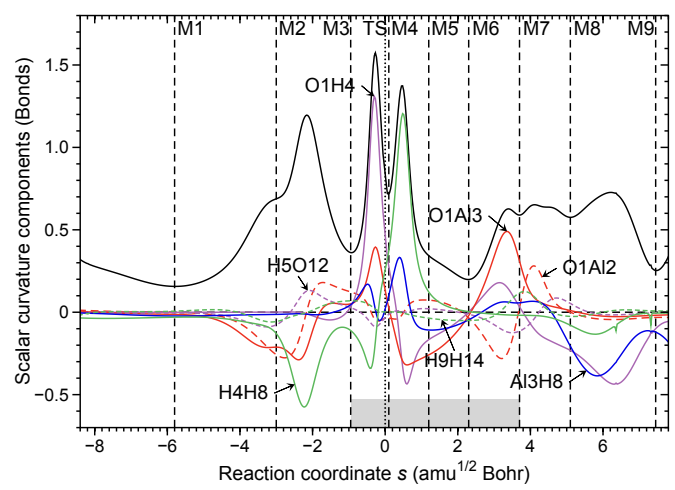

(i) Reaction tR7

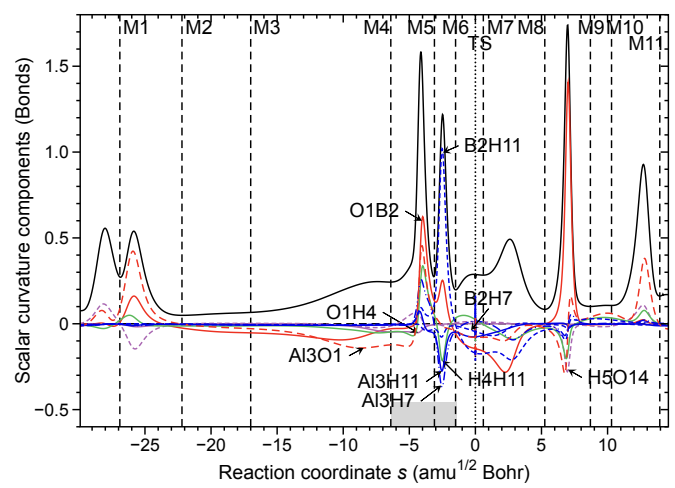

(k) Reaction tR9

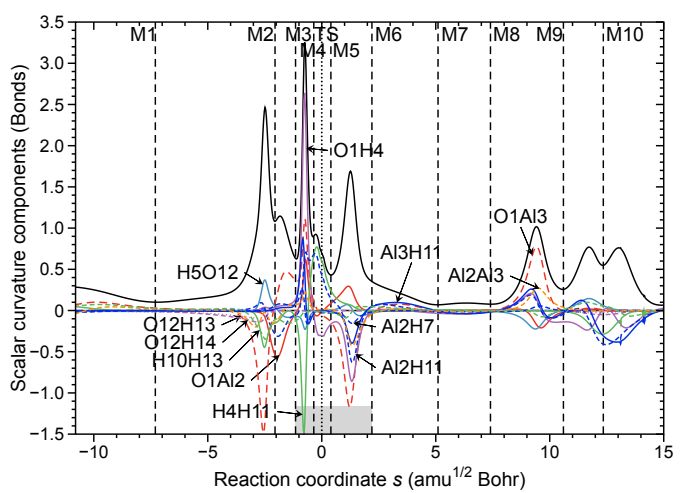

(h) Reaction tR6, Step 2

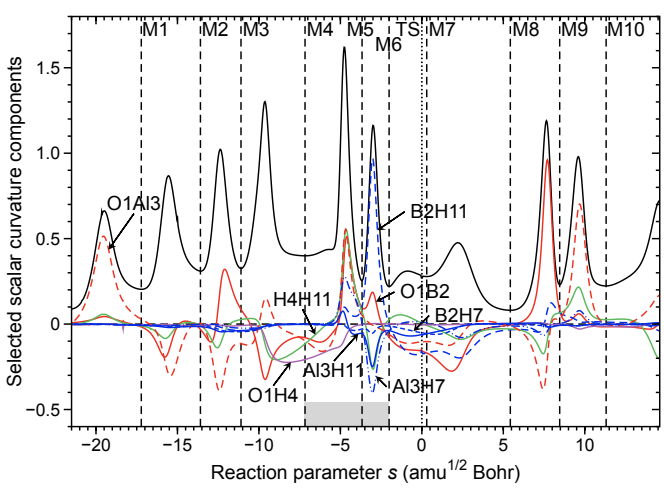

(j) Reaction tR8

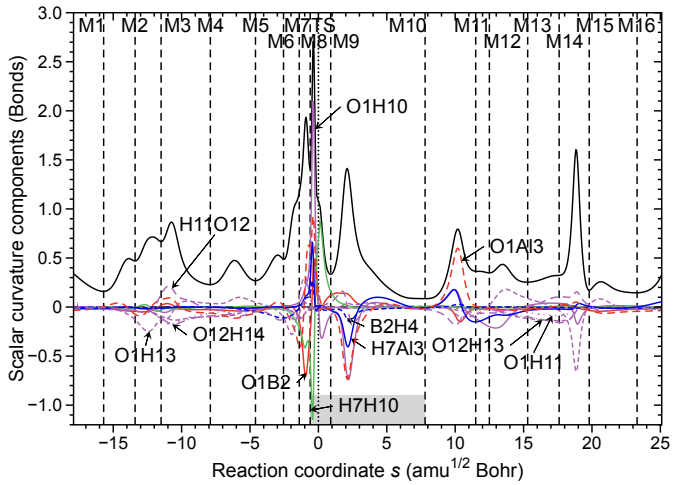

(1) Reaction tR10 


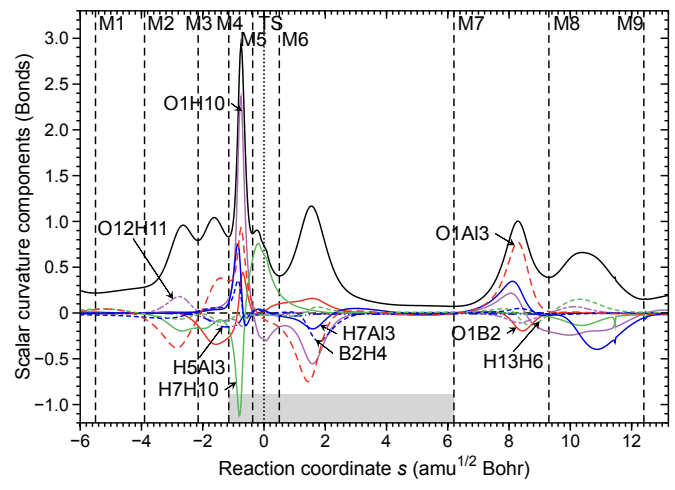

(m) Reaction tR11

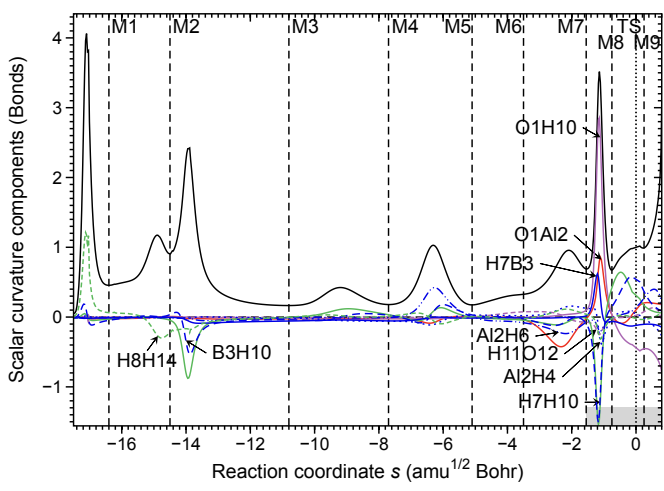

(n) Reaction tR12

Fig. S4: Scalar curvature as a function of the reaction path parameter $s$ (solid black line) for the hydrogen release in the presence of two-water molecules for tR1-tR12. The decomposition of the scalar curvature into the most important bond components is given. The borders of the reaction phases are indicated by vertical dashed lines at curvature minima M1, M2, M3, etc. The TS at $s=0 \mathrm{amu}^{1 / 2} \mathrm{~b}$ is also indicated by a vertical dotted line. The chemical phases are highlighted with a gray bar. 


\section{Scalar curvature decomposition into selected angle and dihedral components}

\subsection{Decomposition into angle components for reactions oR 1-oR9}

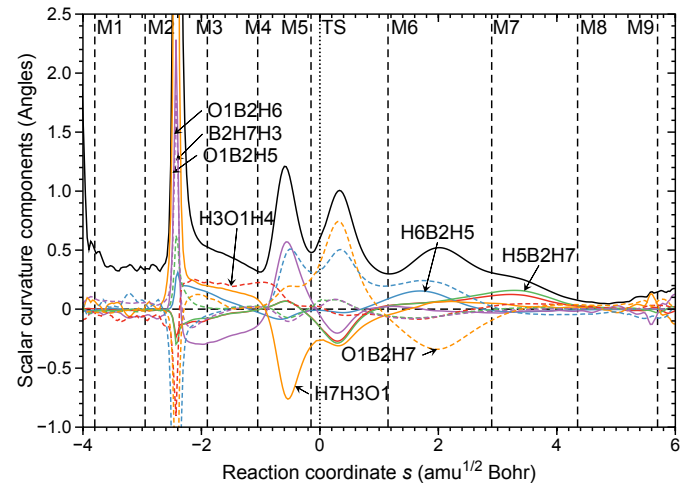

(a) Reaction oR1, PCM

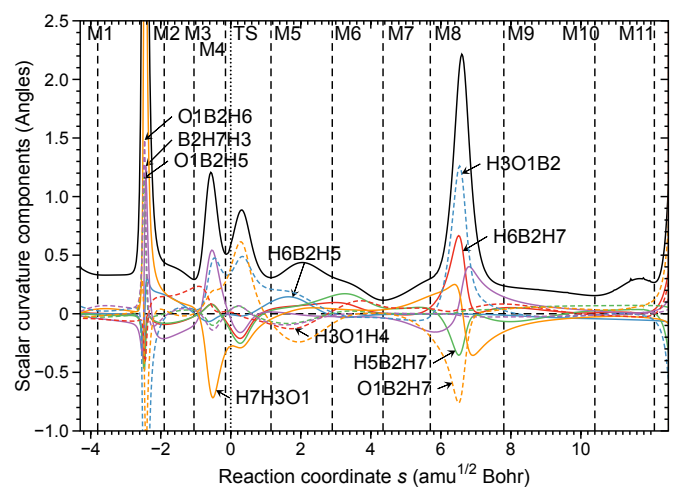

(b) Reaction oR1

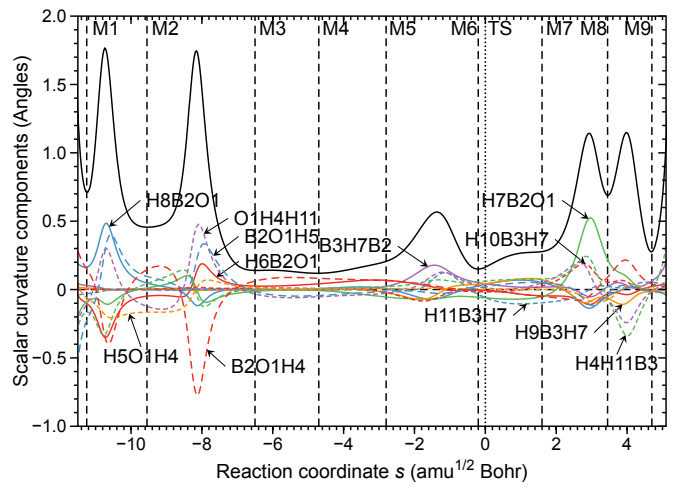

(d) Reaction oR3

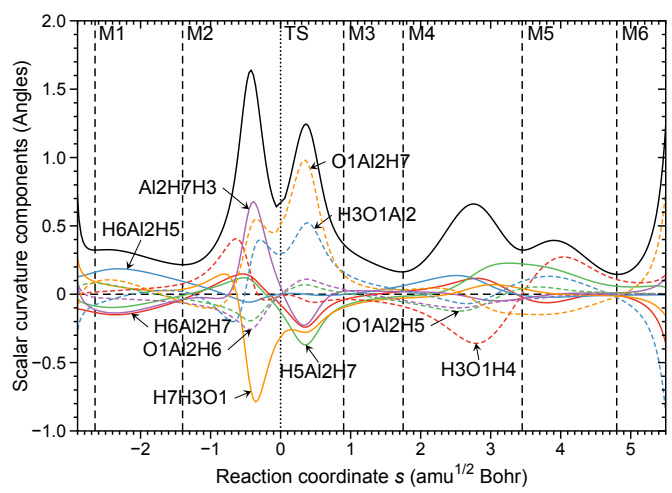

(c) Reaction $\mathbf{0 R 2}$

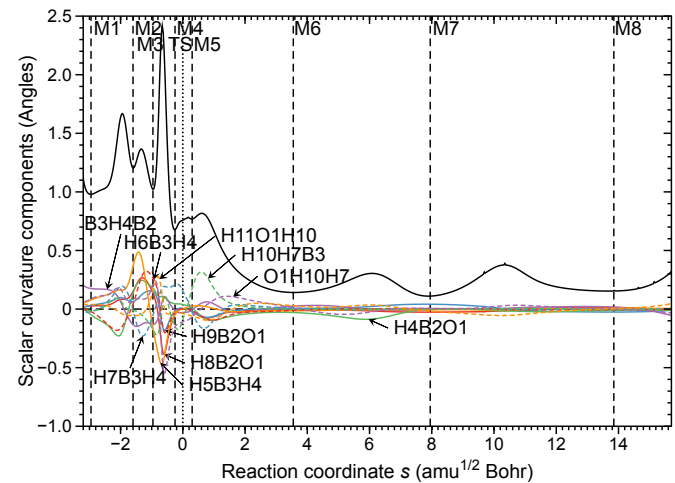

(e) Reaction oR4 


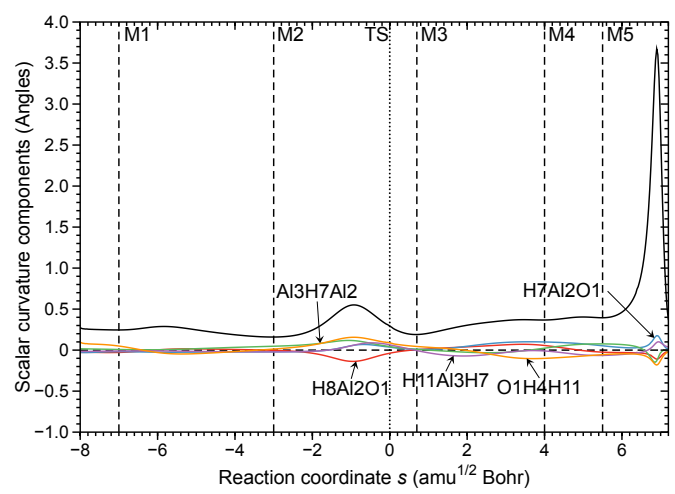

(f) Reaction oR5, Step 1

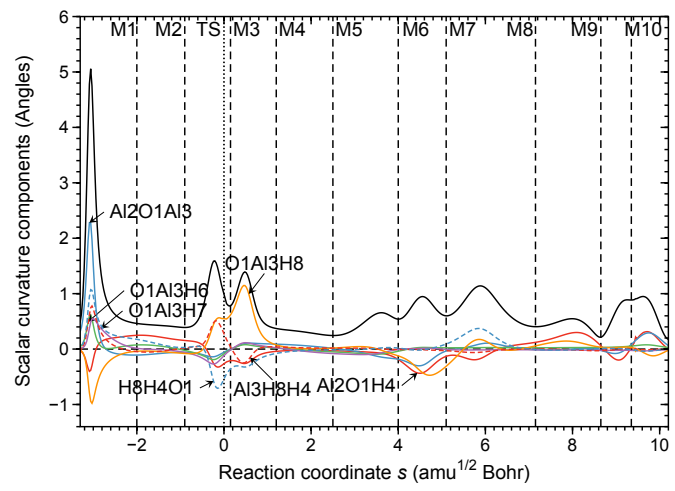

(h) Reaction oR6

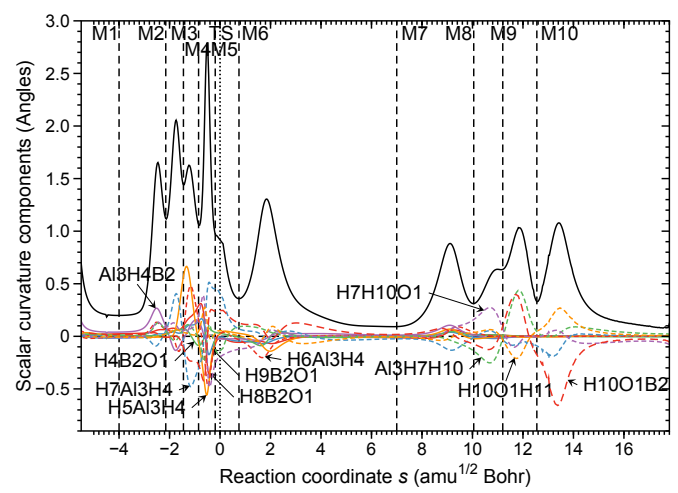

(j) Reaction oR8

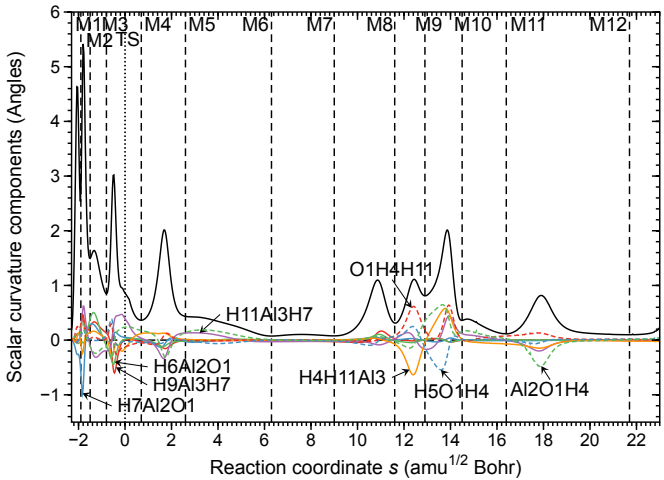

(g) Reaction oR5, Step 2

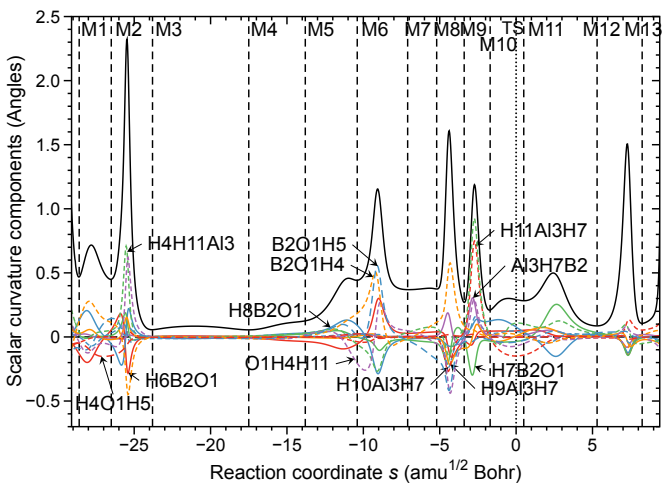

(i) Reaction oR7

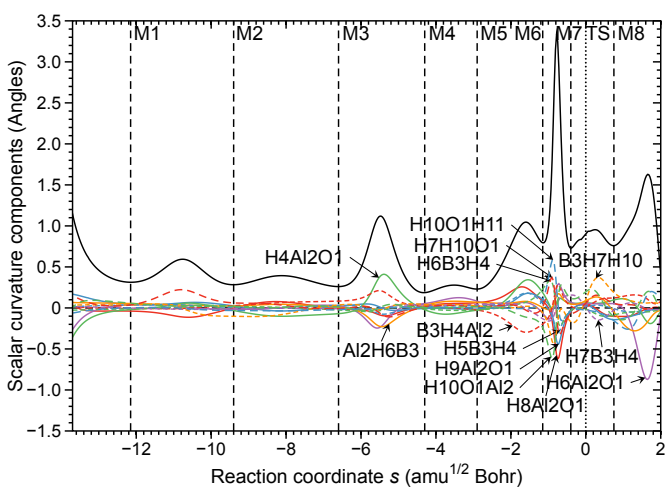

(k) Reaction oR9

Fig. S5: Scalar curvature as a function of the reaction path parameter $s$ (solid black line) for the hydrogen release in the presence of one-water molecule for oR1-oR9. The decomposition of the scalar curvature into the most important angle components is given. The borders of the reaction phases are indicated by vertical dashed lines at curvature minima M1, M2, M3, etc. The TS at $s=0 \mathrm{amu}^{1 / 2} \mathrm{~b}$ is also indicated by a vertical dotted line. 


\subsection{Decomposition into dihedral components for reactions oR $1-o R 9$}

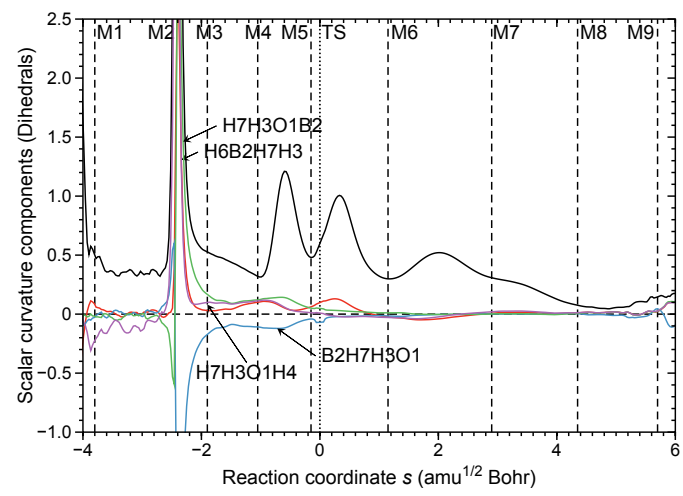

(a) Reaction oR1, PCM

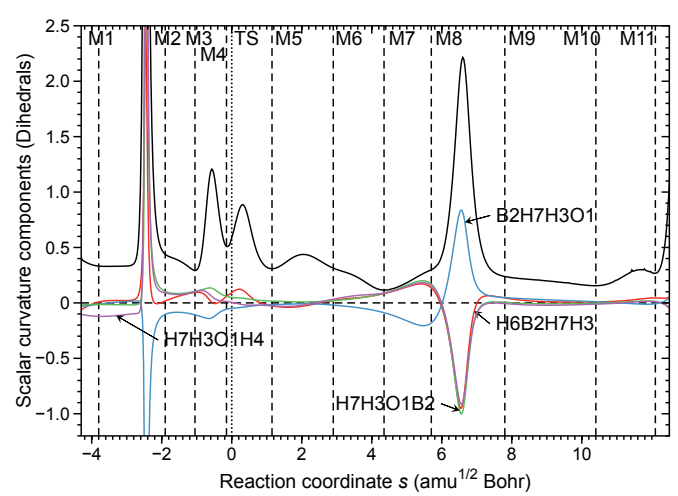

(b) Reaction $\mathbf{o R} 1$

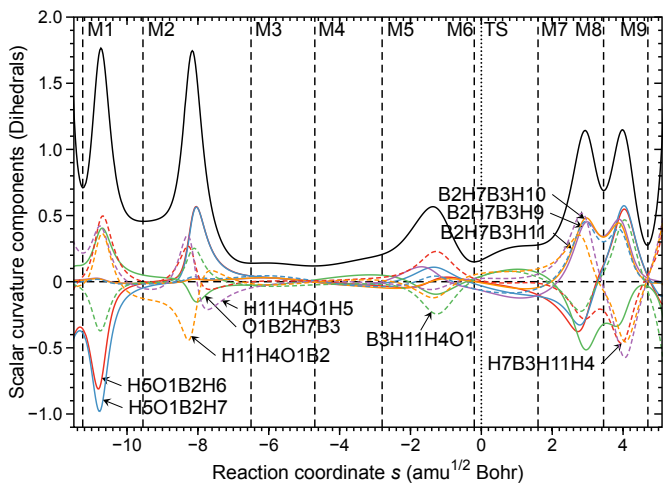

(d) Reaction $\mathbf{0 R 3}$

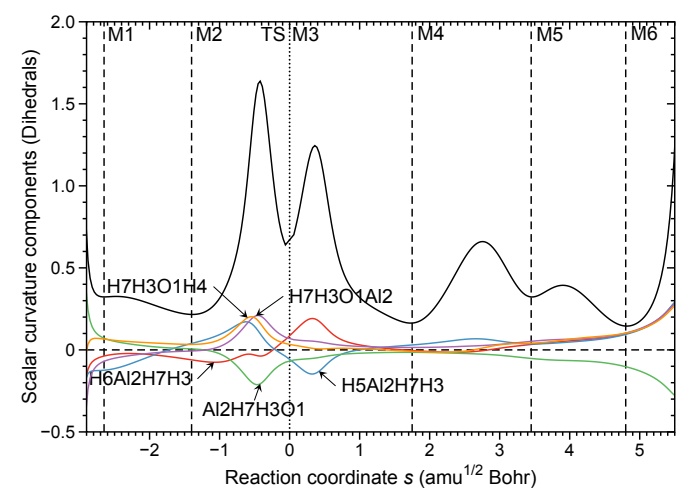

(c) Reaction $\mathbf{O R 2}$

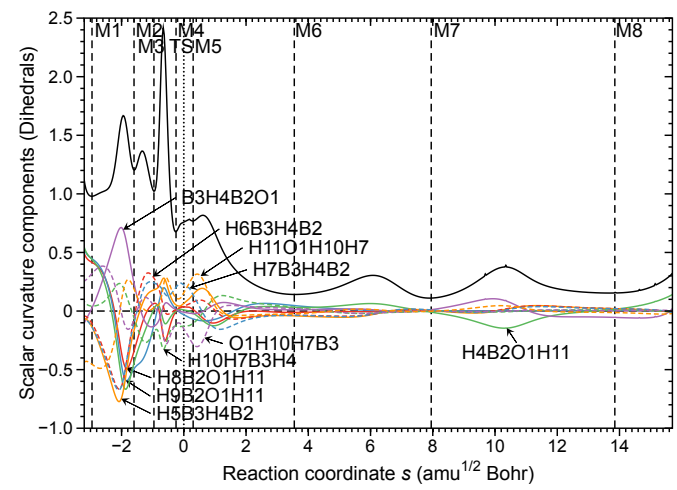

(e) Reaction oR4 


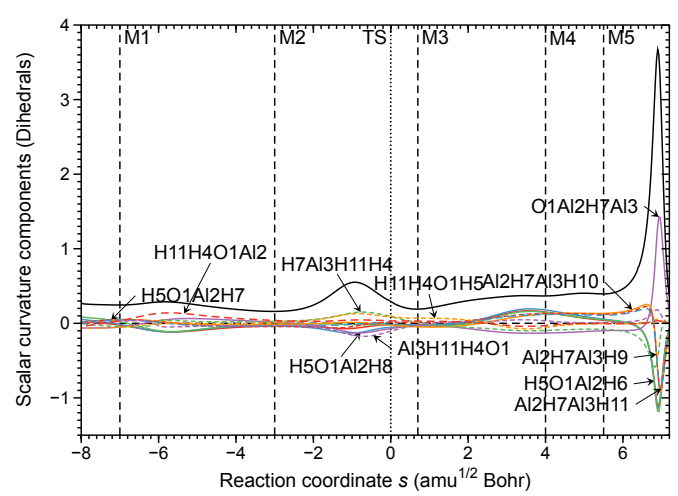

(f) Reaction oR5, Step 1

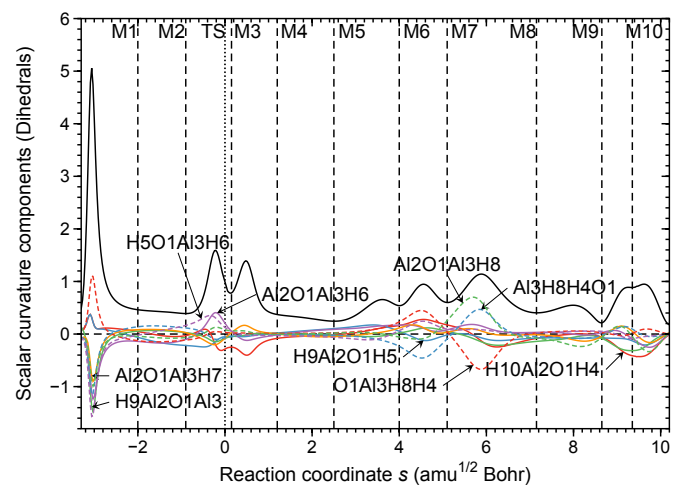

(h) Reaction oR6

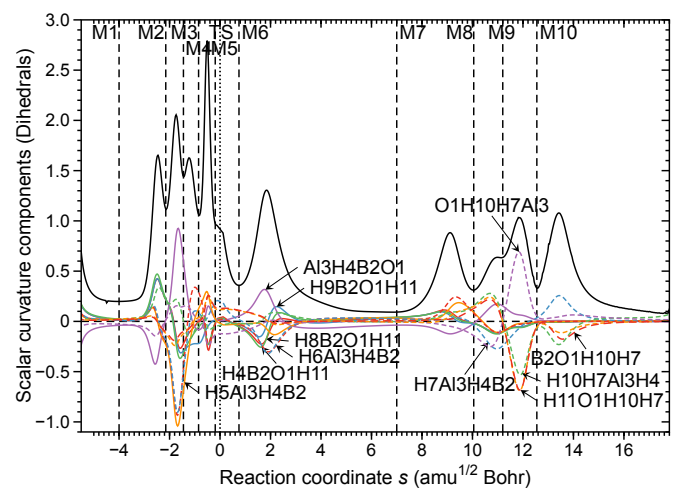

(j) Reaction oR8

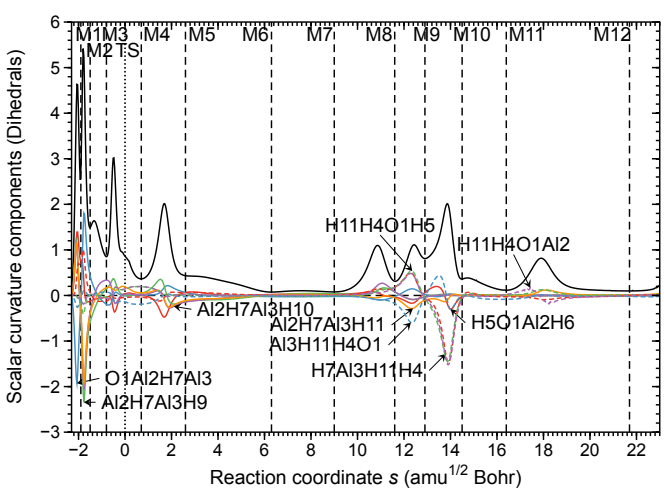

(g) Reaction oR5, Step 2

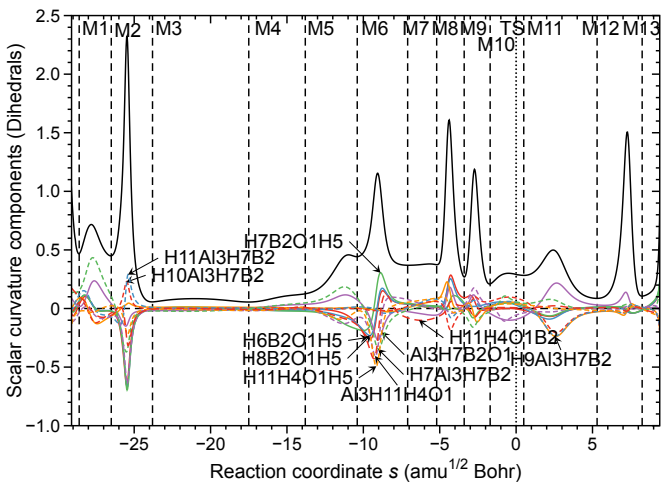

(i) Reaction $\mathbf{o R 7}$

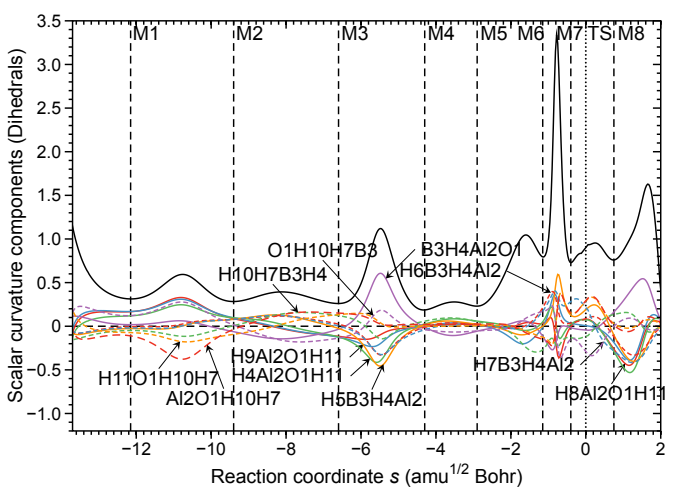

(k) Reaction oR9

Fig. S6: Scalar curvature as a function of the reaction path parameter $s$ (solid black line) for the hydrogen release in the presence of one-water molecule for oR1-oR9. The decomposition of the scalar curvature into the most important dihedral components is given. The borders of the reaction phases are indicated by vertical dashed lines at curvature minima M1, M2, M3, etc. The TS at $s=0 \mathrm{amu}^{1 / 2} \mathrm{~b}$ is also indicated by a vertical dotted line. 


\subsection{Decomposition into angle components for reactions $t R 1-t R 12$}

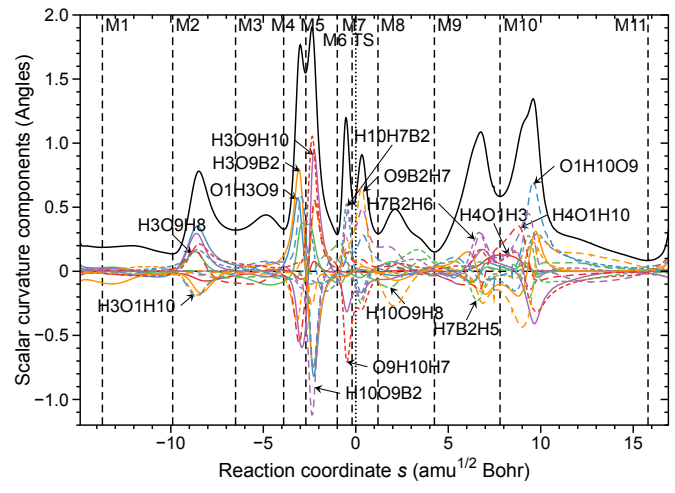

(a) Reaction tR1

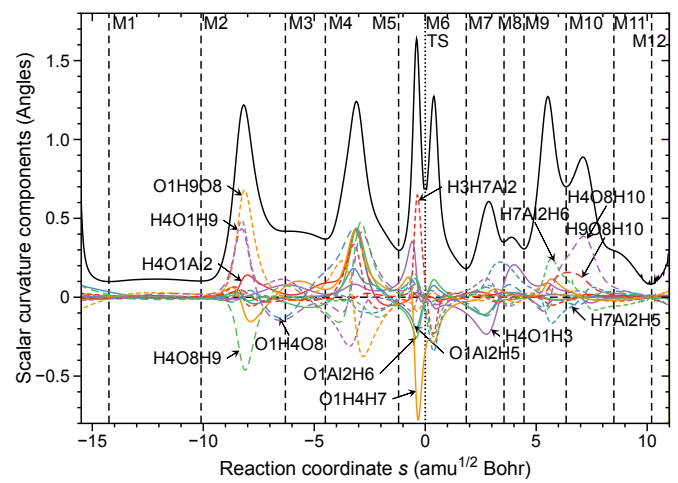

(c) Reaction tR3

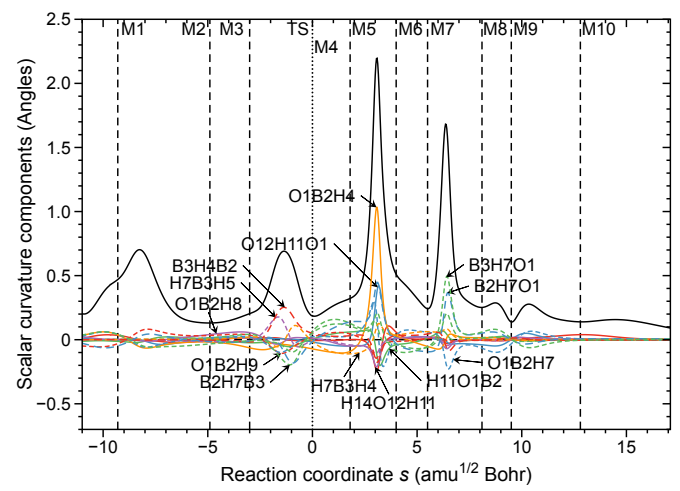

(e) Reaction tR5, Step 1

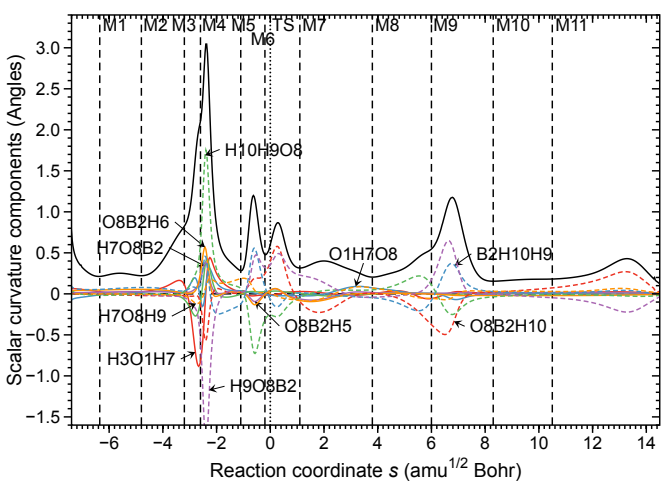

(b) Reaction tR2

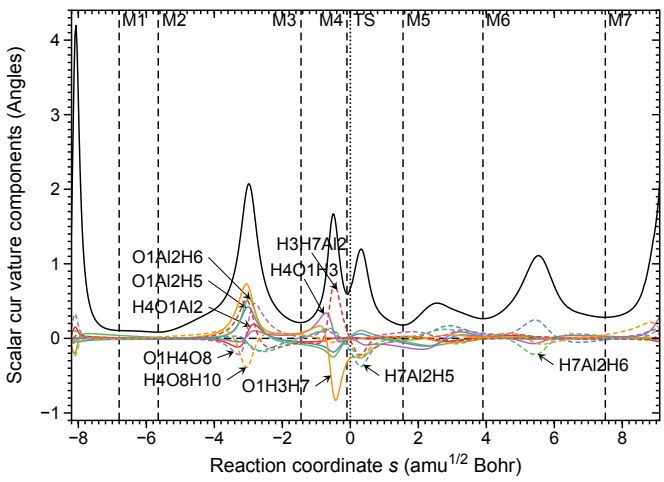

(d) Reaction tR4

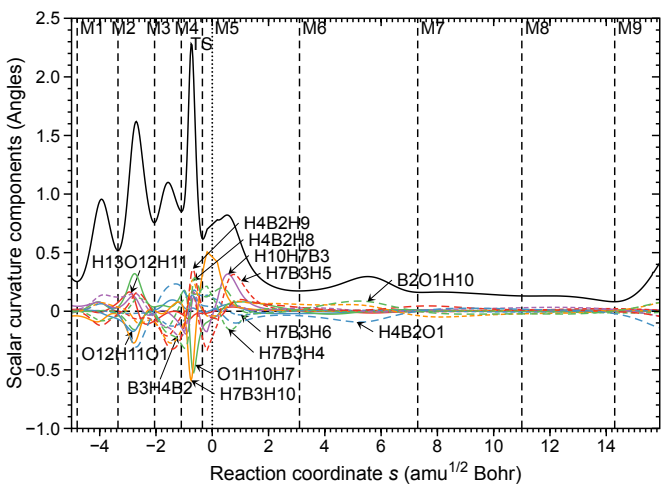

(f) Reaction tR5, Step 2 


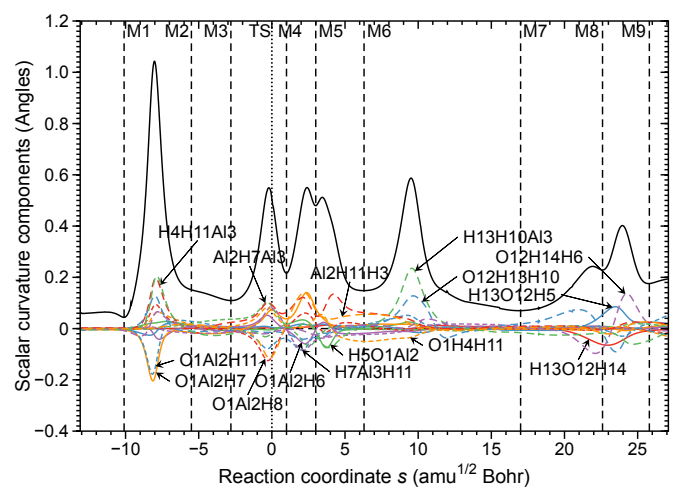

(g) Reaction tR6, Step 1

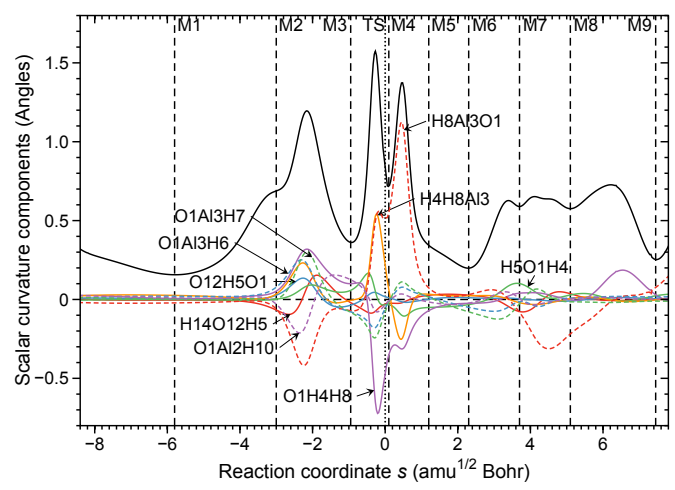

(i) Reaction tR7

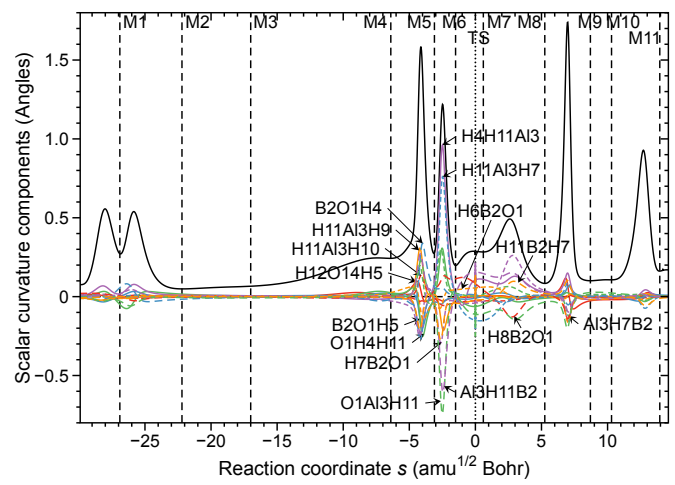

(k) Reaction tR9

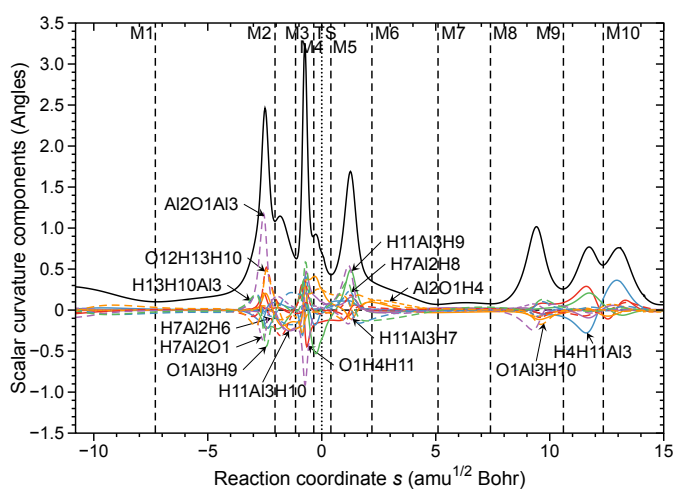

(h) Reaction tR6, Step 2

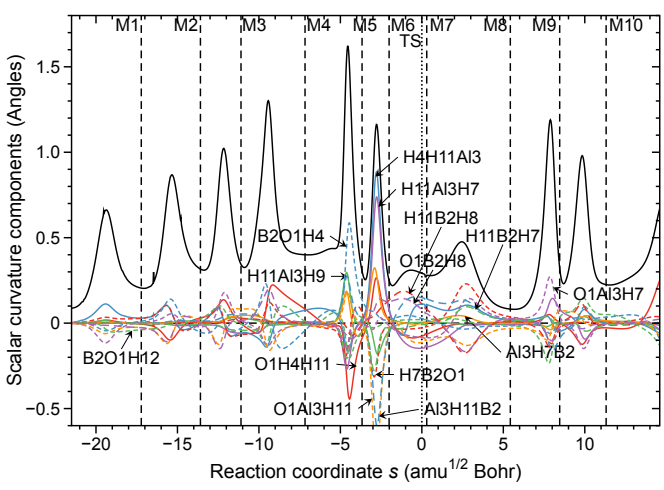

(j) Reaction tR8

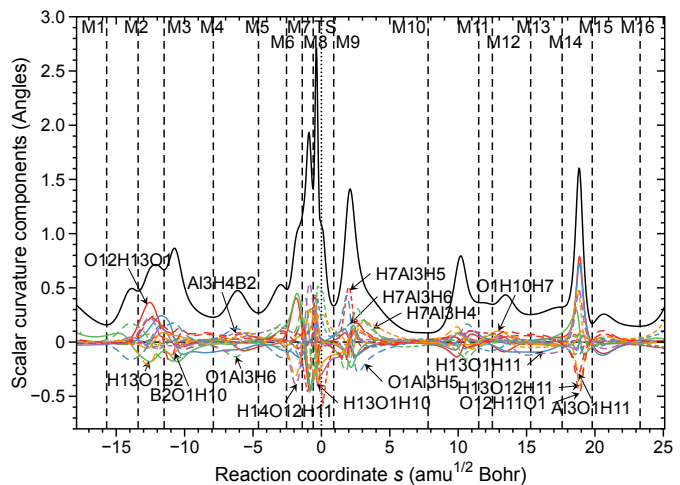

(1) Reaction tR10 


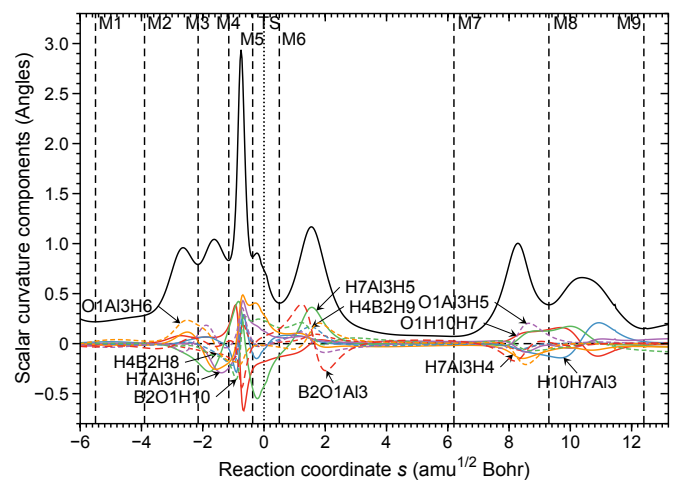

(m) Reaction tR11

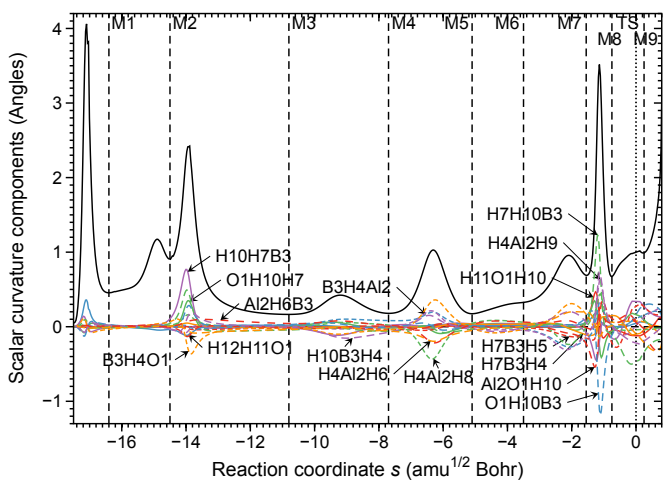

(n) Reaction tR12

Fig. S7: Scalar curvature as a function of the reaction path parameter $s$ (solid black line) for the hydrogen release in the presence of two-water molecules for tR1-tR12. The decomposition of the scalar curvature into the most important angle components is given. The borders of the reaction phases are indicated by vertical dashed lines at curvature minima M1, M2, M3, etc. The TS at $s=0 \mathrm{amu}^{1 / 2} \mathrm{~b}$ is also indicated by a vertical dotted line. 


\subsection{Decomposition into dihedral components for reactions $t R 1-t R 12$}

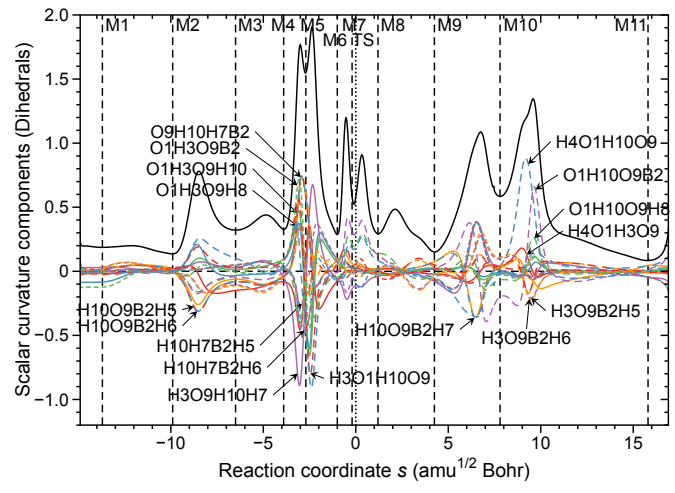

(a) Reaction tR1

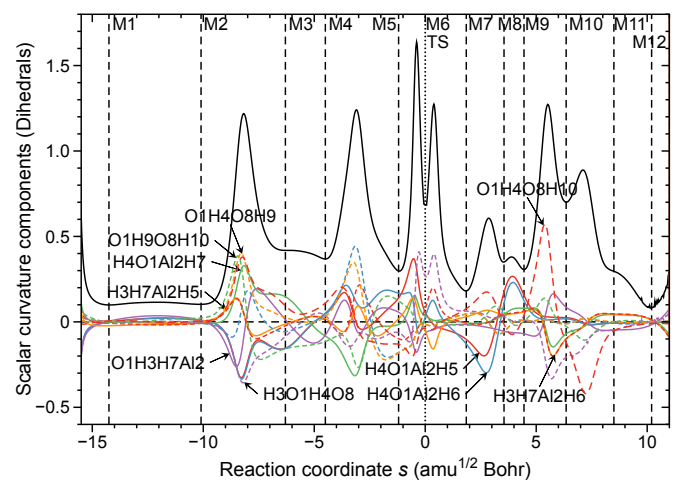

(c) Reaction tR3

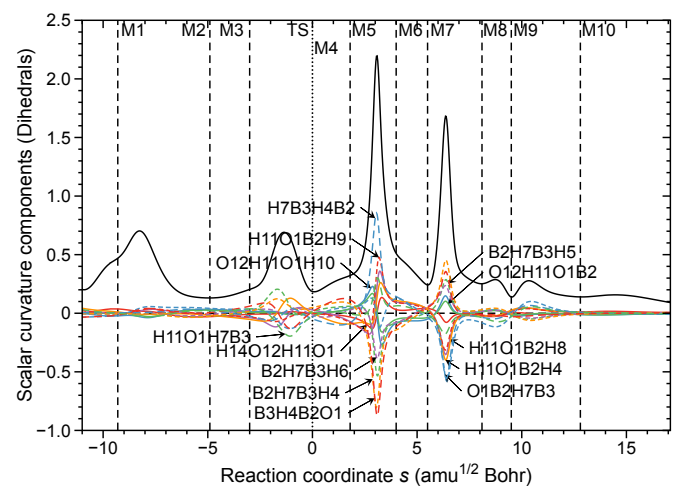

(e) Reaction tR5, Step 1

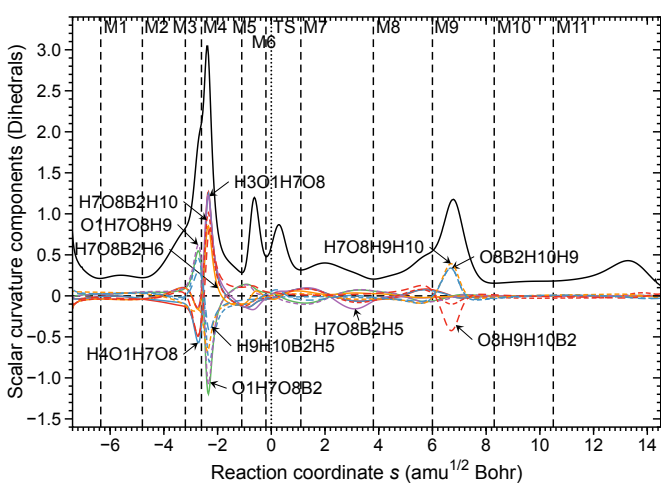

(b) Reaction tR2

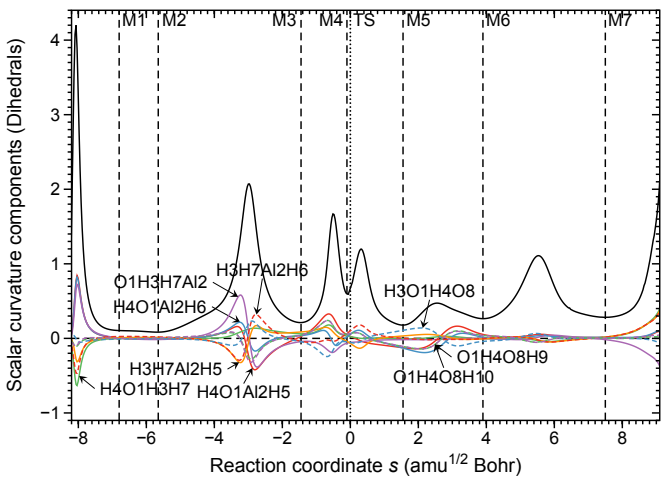

(d) Reaction tR4

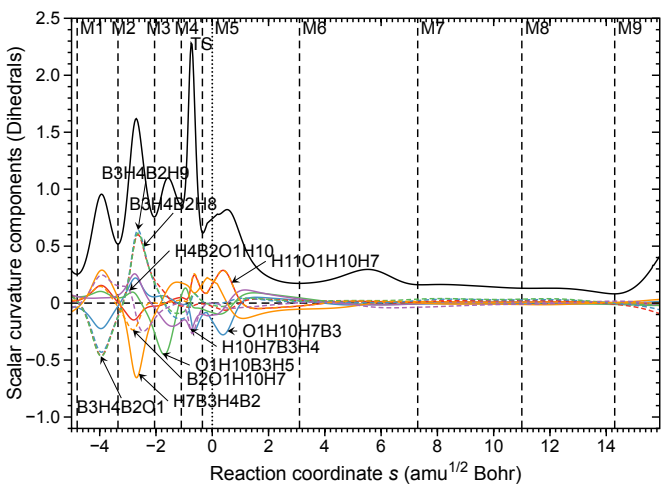

(f) Reaction tR5, Step 2 


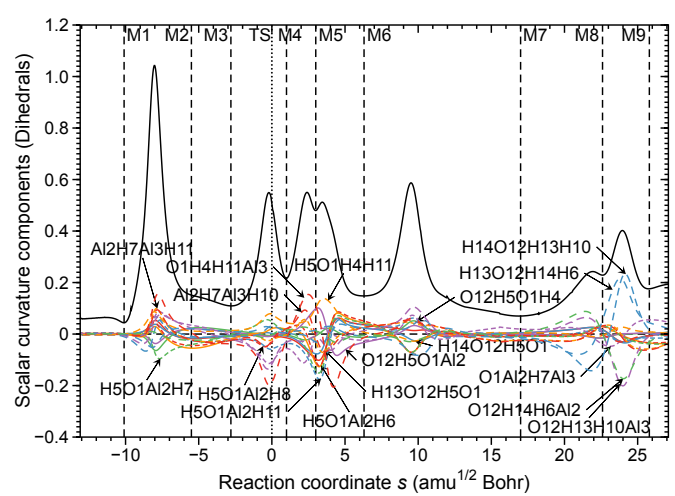

(g) Reaction tR6, Step 1

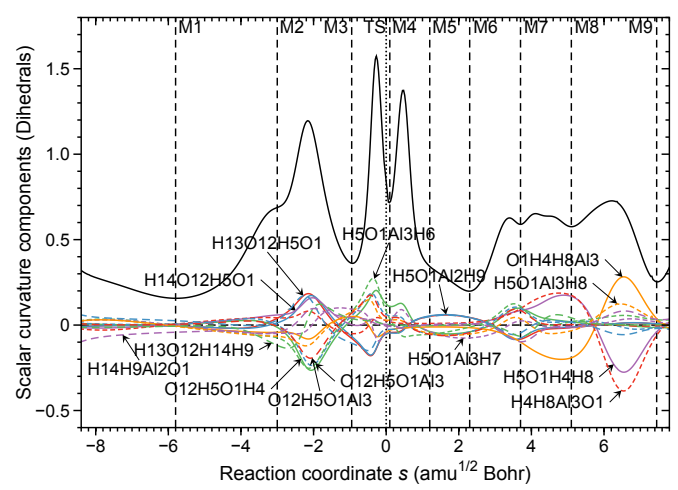

(i) Reaction tR7

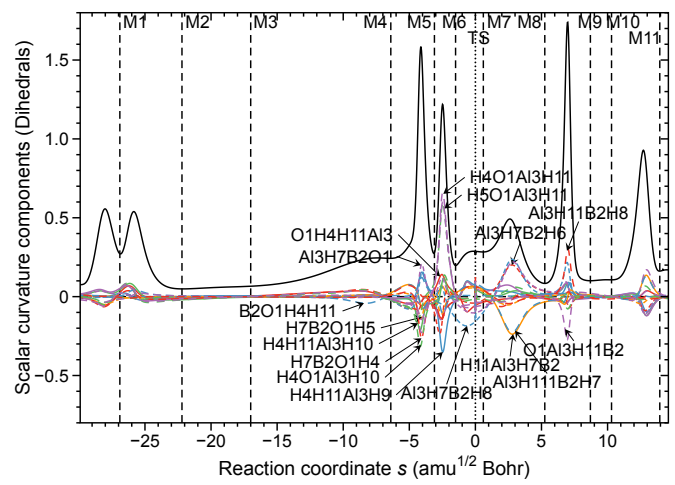

(k) Reaction tR9

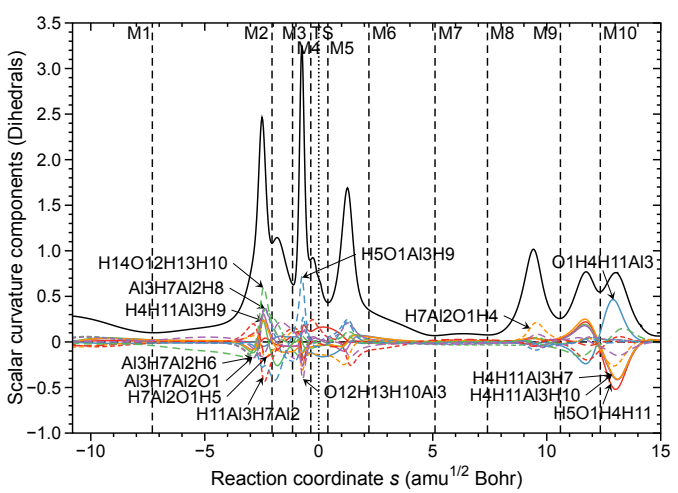

(h) Reaction tR6, Step 2

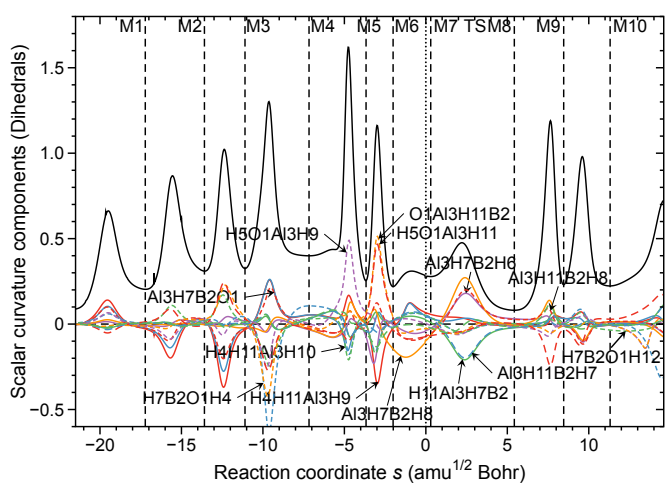

(j) Reaction $\mathbf{t R 8}$

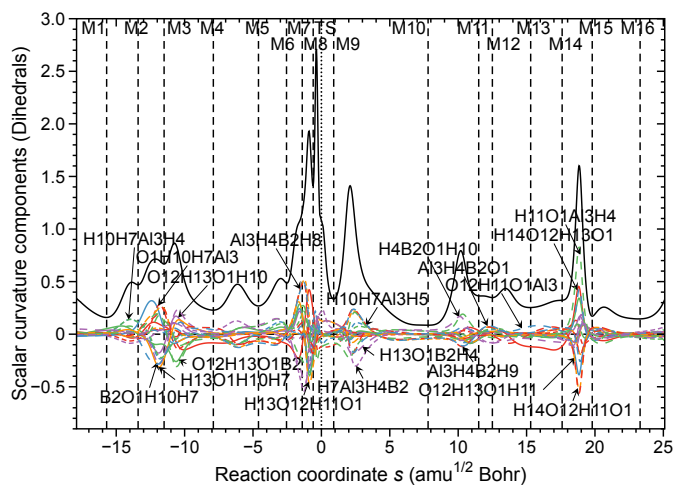

(1) Reaction tR10 


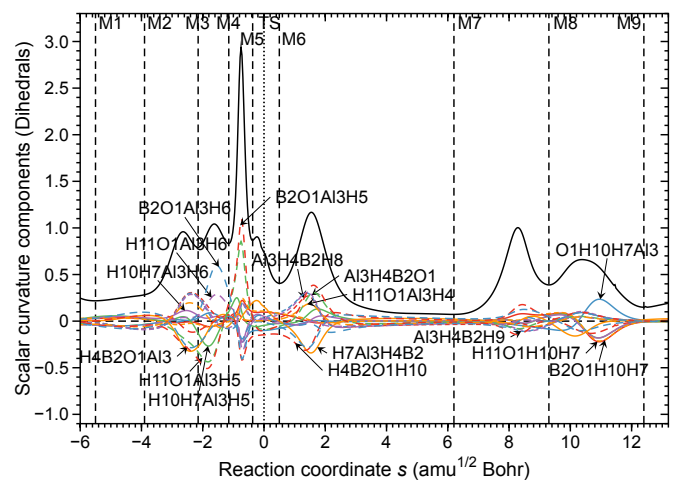

(m) Reaction tR11

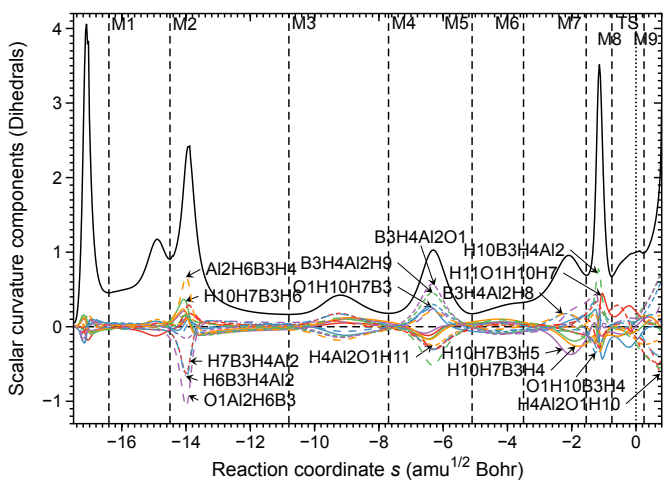

(n) Reaction tR12

Fig. S8: Scalar curvature as a function of the reaction path parameter $s$ (solid black line) for the hydrogen release in the presence of two-water molecules for tR1-tR12. The decomposition of the scalar curvature into the most important dihedral components is given. The borders of the reaction phases are indicated by vertical dashed lines at curvature minima M1, M2, M3, etc. The TS at $s=0 \mathrm{amu}^{1 / 2} \mathrm{~b}$ is also indicated by a vertical dotted line. 


\section{Correlations between activation energy $\Delta E^{a}$ and the cleaving $\mathrm{OH}$ and $\mathrm{XH}(\mathrm{X}=\mathrm{B}, \mathrm{Al})$ bonds for reactions tR1-tR12}
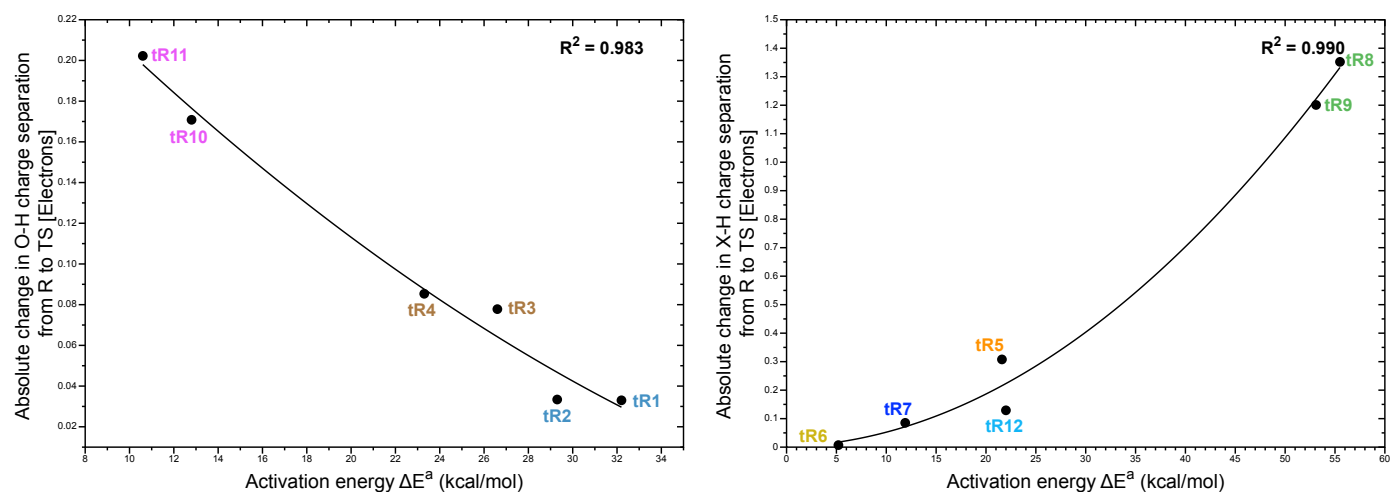

(a) Correlation between activation energy $\Delta E^{a}$ and ab- (b) Correlation between activation energy $\Delta E^{a}$ and absolute change in the charge separation between $\mathrm{O}$ and $\mathrm{H}$ solute change in the charge separation between $\mathrm{X}(\mathrm{X}=\mathrm{B}$, atoms in the cleaving $\mathrm{OH}$ bond going from $\mathrm{R}$ to $\mathrm{TS}$.

$\mathrm{Al}$ ) and $\mathrm{H}$ atoms in the cleaving $\mathrm{XH}$ bond going from $\mathrm{R}$ to TS.

Fig. S9: Correlations between activation energy $\Delta E^{a}$ and the cleaving $\mathrm{OH}$ and $\mathrm{XH}(\mathrm{X}=\mathrm{B}, \mathrm{Al})$ bonds for reactions tR1-tR12.

\section{Local mode analysis of B(Al)-H cleavage for reactions tR1-tR12}

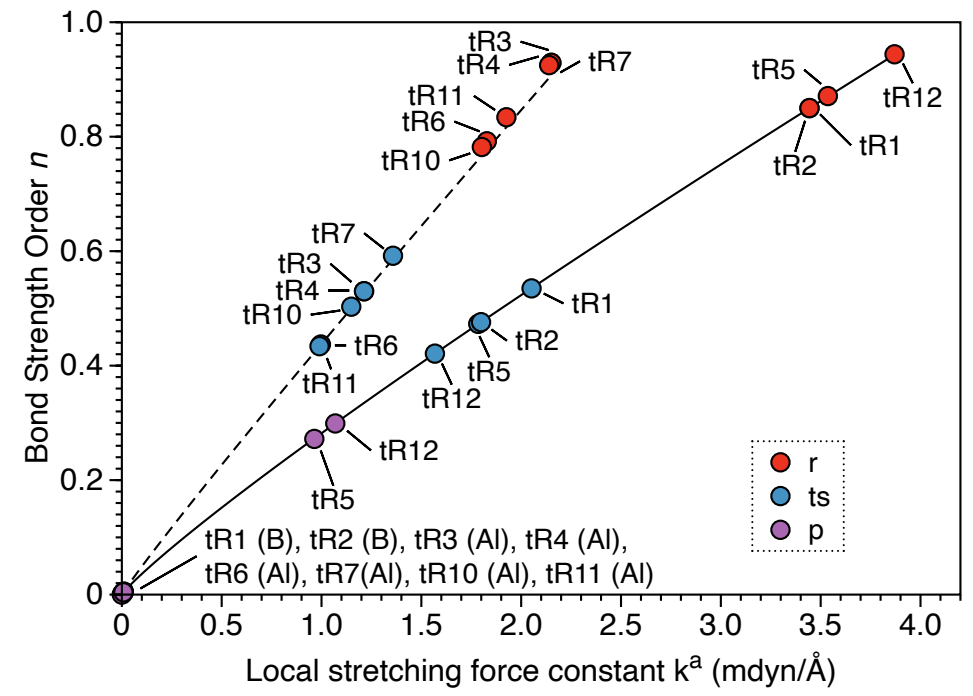

Fig. S10: Power relationship between the $B S O n$ and $\mathrm{k}^{a}$ values for B-H and Al-H bonds at R, TS, and P. Otherwise not stated (in parentheses) solid and dash curves represent B-H and Al-H bonds, respectively. 


\section{Puckering coordinates}

A six-membered ring such as cyclohexane has 6-3 $=3$ puckering coordinates, which split up into the pseudorotational coordinate pair $\left(\mathrm{q}_{2}, \Phi_{2}\right)$ describing the pseudorotation of boat and twistboat forms and a single "crown" puckering amplitude q3, which describes the chair form (positive q3) and the inverted chair form (negative q3). For pseudorotational cycles, the puckering amplitude is always positive.

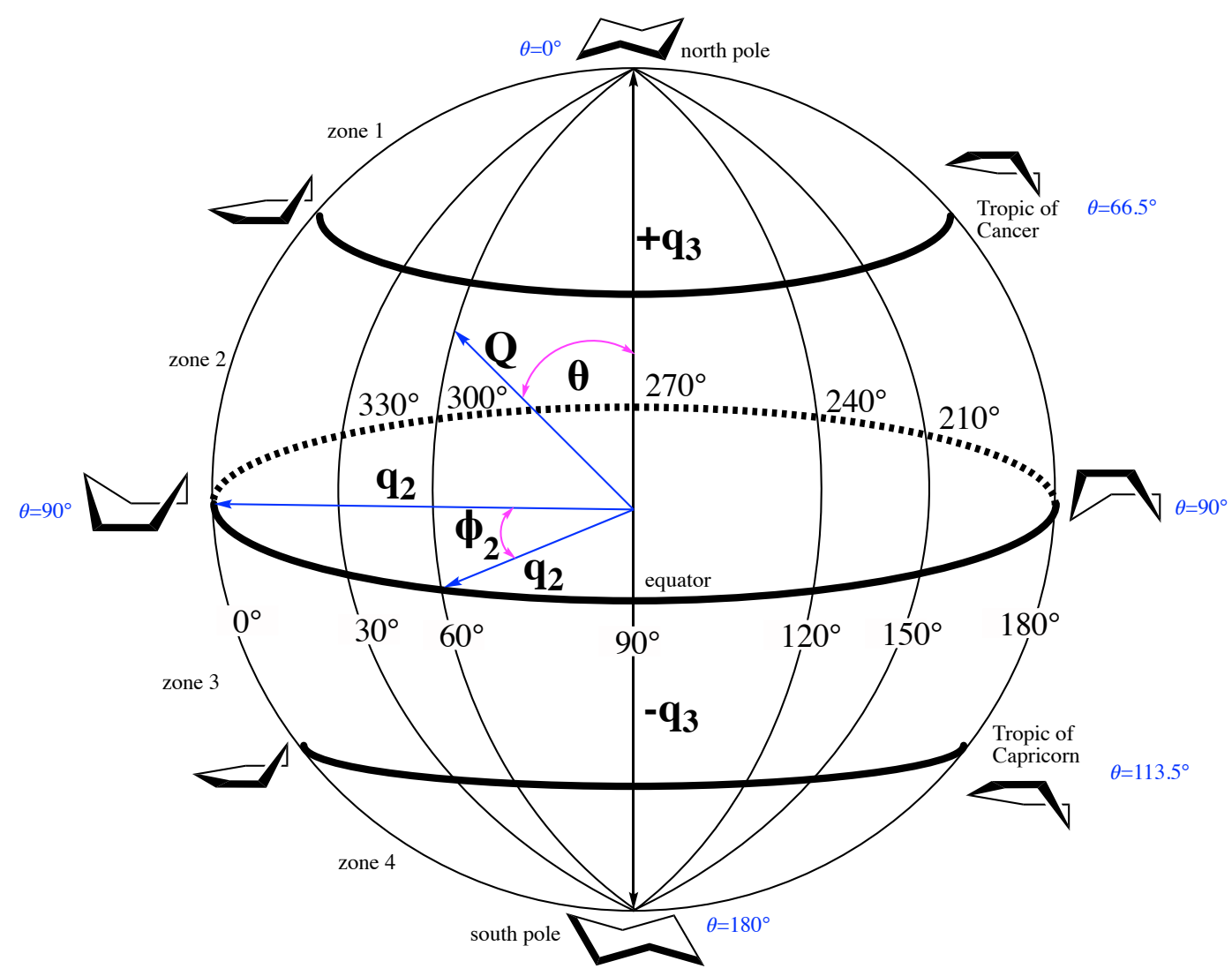

Fig. S11: Conformational globe of a puckered cyclohexane. Puckering coordinates $\mathrm{q}_{2}, \Phi_{2}, \mathrm{q}_{3}$ that span the globe as well as some distinct longitudes and latitudes $(\theta)$ are shown. Distinct ring forms are shown in steps of $30^{\circ}$ along the equator, the tropic of Cancer, and the tropic of Capricorn. 


\section{Coordinates of the stationary points for the reactions oR1-oR9 and tR1-tR12}

\subsection{Coordinates of oR1-oR9}

oR1 reactant:

O -0.763087000 .019161000 .05124900$

B $0.97578400-0.07324000-0.01896300$

H -1.034816000 .914112000 .29929800$

H $-1.13714300-0.15854500-0.82328200$

H 1.254548000 .191985001 .11327800

H $1.12065800-1.21154100-0.35557200$

H $1.248210000 .75995300-0.83993400$

oR1 TS:

O -0.782728000 .017085000 .12932800$

B $0.81552300-0.107728000 .03321800$

H $-0.046138000 .85832000-0.45391100$

H - $1.19097900-0.57837500-0.51465300$

H 1.322231000 .030277001 .09475100

H $1.16928600-0.95339700-0.72343300$

H $0.929815001 .04513900-0.60347000$

oR1 product:

O $-0.493434000 .14926600-0.09821200$

B $0.59929100-0.563951000 .30242600$

H $0.114705002 .62015700-1.09304200$

H - $1.27689000-0.40170500-0.21601400$

H 1.601530000 .047267000 .46591700

H $0.52310700-1.740885000 .47296300$

H $0.322161003 .26671200-1.37477700$

oR2 reactant:

O - $1.29095700-0.01435100-0.11997000$

Al $0.78262100-0.006739000 .04739100$

$\mathrm{H}-1.72847400-0.448510000 .62478900$

$\mathrm{H}-1.702810000 .85190700-0.23946000$

H $1.22992300-0.57889200-1.35985600$

H 0.932427001 .558414000 .29880600

H $0.80498500-0.974757001 .31099200$

oR2 TS:

O - $1.195036-0.008833-0.169856$

Al $0.7149400 .029660-0.014996$

H $-0.767574-0.6479710 .777202$

H - 1.7420970 .7553220 .058054

H $1.452140-0.789797-1.131395$

H 1.2287471 .3849630 .600296

H 0.094855 -1.017440 1.249632

oR2 product:

O - $1.114083000 .08088900-0.26212500$

Al $0.601260000 .05413500-0.04079200$

H -0.00384700 -1.88650000 2.37745200

H - 1.695431000 .738388000 .13016300
H $1.36619800-1.09613900-0.77952200$

H 1.296571001 .158603000 .84099600

H $0.62085700-1.647434002 .68310600$

oR3 reactant:

O - $1.84859500-0.66358000-0.14322800$

B 0.732339001 .142936000 .19838300

B $1.72083000-0.27836800-0.01097200$

H $-2.43889500-0.876238000 .58875100$

H -2.40991100 -0.63523900 -0.92650900

Н 0.390487001 .452921001 .28972400

H 2.032714000 .970733000 .19695000

H $0.431905001 .78255900-0.75270300$

H $2.07258700-0.58444900-1.10089800$

H $2.03119400-0.913888000 .94040400$

H $0.43046900-0.10929100-0.00982400$

oR3 TS:

O - $1.357944-0.317981-0.119277$

B 0.1093660 .8635580 .022315

B $1.529726-0.4154210 .019203$

$\mathrm{H}-1.083843-1.2133700 .134834$

$\mathrm{H}-2.047586-0.0438550 .504355$

$\mathrm{H}-0.3262021 .3395751 .029347$

H 1.3768960 .7634400 .441702

Н $0.0361701 .479306-0.984816$

H $2.544427-0.292817-0.591984$

H $1.511267-1.1012620 .998965$

H $0.656961-0.627855-0.785778$

oR3 product:

O - $1.14264100-0.26157500-0.02402500$

B -0.15167300 0.997830000 .00249700

B $1.50771300-0.59104100-0.00004600$

H $-0.66211500-0.94930000-0.53297700$

$\mathrm{H}-1.26368100-0.618302000 .87044700$

H - 0.466477001 .635416000 .95689500

H 1.031393000 .624295000 .28107000

H $-0.183431001 .48102700-1.07831700$

H $2.65876700-0.28477300-0.05684400$

H $1.16793200-1.222859000 .96582600$

H $1.03901000-0.95781400-1.05158000$

oR4 reactant:

O $-1.06078500-0.49524300-0.06400200$

B -0.35722400 $0.94388900-0.01183700$

B $1.59394800-0.262418000 .11399300$

H $0.895254000 .83129200-0.19997500$

H 2.647545000 .276806000 .25932600

H $1.13593400-0.738270001 .12578200$ 
H $1.46713500-0.93360100-0.87645400$ H -0.569944001 .389936001 .06447200$ H $-0.727115001 .51881500-0.98607200$ H $-0.48571600-1.076386000 .47865100$ H - $1.03742400-0.85301000-0.96591200$

oR4 TS:

O $-0.97063100-0.61155000-0.08724000$ B -0.66529600 0.84150900 -0.00108300 B $1.54121700-0.017620000 .07916300$ H $0.759869000 .95509800-0.05814200$ H $2.581174000 .28249700-0.41527900$ H $1.52640300-0.447193001 .18807300$ H $1.14230200-0.91182100-0.78133500$ $\mathrm{H}-0.898132001 .358810001 .04336600$ H -0.85890600 $1.40610300-1.02821500$ H $0.30002600-0.88582400-0.42449200$ H - $1.16729800-0.984708000 .78354300$

oR4 product: O $-0.753561000 .03118000-0.16653900$ B - 1.803914000 .839414000 .17225700 B $2.39801700-0.74243300-0.01299300$ H $1.91552600-1.27667900-0.96342100$ H $3.22701200-1.378637000 .56749300$ H $2.567405000 .43586000-0.03627200$ H $1.63739700-1.063239001 .12046300$ H -2.09801100 0.867414001 .32005600 H -2.37386000 $1.46703300-0.66261400$ H $1.13964800-0.654218000 .66784500$ H -0.54543200 0.04820700 - 1.11022000

oR5, Step 1 reactant: O 2.21550400 0.86100900 -0.18923300 Al $0.63012500-0.577211000 .06200800$ Al - 1.949428000 .035502000 .05476200 H 2.33993600 1.29301300-1.04452600 H 2.387428001 .511256000 .50449600 H $1.10909300-0.818067001 .54375800$ H - $1.04013300-1.354280000 .22269900$ H $1.02124600-1.22179000-1.32166300$ H -2.66792100 0.11507800 - 1.34572500 H -2.583152000 .504669001 .41938800$ H -0.40769500 $0.80794300-0.10133600$

oR5, Step 1 TS:

O $1.691110000 .95080100-0.11949200$ Al $0.76697900-0.848006000 .03277600$ Al - 1.790182000 .221898000 .01381900 H $1.195881001 .70871700-0.46407500$ H 2.252146001 .221316000 .62081600 H $1.70084300-1.173677001 .27736900$ H -0.76850400 -0.94736100 0.77150500 H $0.86846200-1.64479500-1.31546500$
H $-2.94062800-0.56546200-0.71888100$ $\mathrm{H}-2.023471001 .332343001 .11902400$ H $-0.511966000 .60191400-0.94009300$

oR5, Step 1 product: O $1.368617000 .90985400-0.17364100$ Al $1.03768400-0.999499000 .10276400$ Al -1.80785800 $0.42221900-0.01369700$ H $0.492708001 .33737000-0.44748000$ H 1.754537001 .421123000 .55153000 H $1.55708000-1.252797001 .56675300$ H $-0.62093800-0.868175000 .06700100$ H $1.51234200-1.74639200-1.19304400$ H -2.94151700 -0.18238500 -0.92729200 H - 2.00451000 0.78245100 1.51481300 H - $0.851508001 .52372400-0.76097000$

oR5, Step 2 reactant: O $1.028867001 .29018800-0.08997600$ Al 1.29124500 -0.64734300 0.00313600 Al - $1.85104600-0.139900000 .03262600$ H $0.057046001 .45835900-0.31857900$ H 1.267803001 .825835000 .67956600 H $1.90885900-0.864684001 .43439900$ H -0.33135300 - $1.01667300-0.01448500$ H $1.92551300-1.09706700-1.35984600$ H -2.78104900 -0.97113700 -0.93149400 H - 2.09780600 0.004490001 .58923400 H - $1.290872001 .26075800-0.60823200$

oR5, Step 2 TS: O $0.956065001 .18943100-0.08025800$ Al 1.31566000 -0.62791700 0.00221100 Al - $1.77116200-0.152777000 .03629800$ H $-0.297121001 .32025100-0.38996600$ H 1.242913001 .777208000 .63115900 H $1.77219400-1.044543001 .45192500$ H $-0.36790900-1.05068600-0.14670900$ H $1.93468700-1.17594300-1.33489100$ H -2.85084300 -0.67490100 -0.97840100 $\mathrm{H}-1.980079000 .157995001 .56359800$ H - $1.180840001 .32418700-0.65526100$

oR5, Step 2 product: O $0.086996000 .77764600-0.47582300$ Al $1.31695200-0.522563000 .11865200$ Al - $1.39904100-0.186464000 .17142100$ H $0.467749003 .93181500-0.95373000$ H $0.205427001 .73301000-0.46504400$ H $2.08912800-0.030740001 .39947400$ H $-0.15218800-1.322705000 .59121900$ H $1.96909700-1.32636300-1.06341300$ H -2.27212400 -0.80151900-0.98101000 $\mathrm{H}-1.978514000 .472626001 .47850400$ 
H $0.488428003 .98418900-0.22020200$

oR6 reactant:

O -0.00282400 0.80571600 -0.02796600

Al - $1.95115500-0.24621700-0.01825300$

Al $1.95114300-0.270801000 .03596800$

H 0.018486001 .412123000 .73390900

H $0.026586001 .36513200-0.82465800$

H $1.49046300-1.76851300-0.11160400$

H $2.459317000 .53248800-1.23624200$

H 2.266216000 .341338001 .46557400

H -2.75371500 1.122104000 .09690100

$\mathrm{H}-1.76623000-0.87641100-1.45545100$

H -1.69598900 -1.07394700 1.30116400

oR6 TS:

O $0.001407000 .70074800-0.04916800$

Al - $1.87588800-0.23269200-0.00999100$

Al $1.84321200-0.25390900-0.02994700$

H 0.617236000 .784071000 .92768100

H $-0.039808001 .55583700-0.51198900$

H $1.65719700-1.80271600-0.02859100$

H $2.774934000 .63640000-0.92324200$

H 1.549074000 .479625001 .47786000

H -2.72964000 1.109113000 .02933700

H - $1.77428000-1.00317800-1.39417100$

H - $1.64117700-1.039321001 .33566300$

oR6 product:

O $0.064517000 .55403900-0.49747500$

Al - $1.70280200-0.181300000 .09203500$

Al $1.62560200-0.143894000 .04157600$

H $0.81977200-0.147156002 .11594700$

H $0.014709001 .48606700-0.74765900$

H $1.70269300-1.697443000 .04388800$

H 2.786395000 .906124000 .09416100

H 1.473724000 .202027002 .18630000

$\mathrm{H}-2.464150001 .199955000 .32258100$

H - 2.17739500 - $1.12541300-1.09188000$

H -1.11287800 -0.91105000 1.39491100

oR7 reactant:

O -1.41797100 - 1.058435000 .03501900

B $0.684912001 .92093700-0.00868700$

Al $0.54630500-0.29381200-0.01760500$

H - $1.99939500-0.96887600-0.73186900$

H - $1.95328400-0.978767000 .83582200$

H 0.716727002 .519438001 .02303500

H $1.659020001 .14676800-0.04130100$

H $0.657596002 .53162500-1.03336900$

H $0.79754500-0.88845600-1.45305300$

H $0.87984400-0.905019001 .39393500$

H -0.361391001 .223374000 .01723000$
oR7 TS:

O $-2.33355700-0.47915300-0.06739900$

B -0.33038500 1.44401500 -0.02031200

Al $1.63372100-0.358722000 .00872300$

H - $1.86301100-1.270244000 .22278700$

H $-3.21477000-0.531826000 .32050900$

H -0.446964001 .515264001 .14968200$

H $0.774888001 .26264700-0.49202200$

H - $1.138220001 .77517400-0.80823000$

H $2.30934600-0.59254900-1.39709600$

H 2.468163000 .105682001 .26338900

H $0.19257600-0.987620000 .26833400$

oR7 product:

O - $1.71459400-0.20727000-0.04797400$

B -0.82106600 1.14255900 0.01688700

Al $1.38486800-0.38811600-0.01197300$

H - $1.08089600-0.929791000 .24013700$

H -2.46266100 -0.17348800 0.56625700

H -0.569364001 .360788001 .16311400$

H $0.162431000 .82051600-0.66082000$

H - $1.405859001 .97812000-0.59446400$

H $1.98738000-0.72513000-1.43160300$

H 2.21800600 0.31919400 1.12086100

H $0.25622600-1.451137000 .49398600$

oR8 reactant:

O - $1.61382800-0.65689600-0.07622300$

B -1.11731100 0.86902200 0.04011300

Al 1.444058000 .007062000 .00782300

H -0.047370000 .813598000 .67892700$

H $2.068762000 .96338300-1.07398800$

H $2.10564100-0.242111001 .42201400$

H $0.64291000-1.29721900-0.55789500$

H - 1.894885001 .435605000 .74131600

H $-0.976181001 .24780100-1.07758800$

H $-0.79058900-1.17067900-0.33254300$

H -1.95075100 -1.00706200 0.76184800

oR8 TS:

O - $1.39203900-0.64027000-0.07886700$

B -1.20640200 0.866340000 .00948000

Al 1.349991000 .017301000 .04576400

H $0.085461001 .12821000-0.03493300$

H $2.576564000 .58291100-0.75407700$

H $1.35537800-0.611632001 .48629300$

H $0.56623800-1.15135600-0.96948300$

$\mathrm{H}-1.550766001 .303817001 .06504300$

$\mathrm{H}-1.582325001 .39543200-0.99005500$

H $-0.27639800-1.04296300-0.60168900$

H - $1.55570800-1.038871000 .78750800$

$\mathbf{O R 8}$ product:

O -0.69311300 -0.64972800 0.02569000 
B - $1.164731000 .85098500-0.15428700$ Al 1.008357000 .097122000 .21486200 H -0.04522700 $1.48556000-0.04193700$ H $1.90069900-0.06905400-1.05906900$ H 1.471487000 .113902001 .71442200 H - $1.06561800-2.34562800-2.97959500$ $\mathrm{H}-1.842377001 .198311000 .76318000$ $\mathrm{H}-1.544315001 .03071700-1.26720500$ H - $1.02071500-1.92294400-2.38004000$ H -1.12619900 -1.07535700 0.77562100

oR9 reactant:

O 0.854298001 .547928000 .07903000

Al $0.36571900-0.49973300-0.04002300$ B - $1.80878800-0.94186300-0.01891900$ H $-0.90881400-1.79844000-0.09380300$ H -2.38406300 -1.09271400 1.01547200 $\mathrm{H}-1.261044000 .188817000 .04070800$ H -2.43047000 -0.96201400 - 1.03713800 H $1.03502100-0.796177001 .35331800$ H $0.97067100-0.61542800-1.48843800$ H $0.676586002 .13803700-0.66553800$ H 0.711639002 .038798000 .89932600

oR9 TS:

O $0.386346001 .20561700-0.08203900$ Al $0.84868500-0.556202000 .00038500$ B - $1.81477800-0.355133000 .06143500$ H $-0.89212000-1.02483100-0.41244600$ $\mathrm{H}-2.82631000-0.75691300-0.41940200$ H - $1.77343300-0.209189001 .24078200$ H - $1.819561000 .79917300-0.55324100$ H $1.20900200-1.076424001 .43898700$ H $1.44925000-1.11617200-1.33736300$ H $-0.992551000 .94419600-0.26135800$ H 0.595939001 .801517000 .64817000

oR9 product: O $0.442959001 .19942100-0.04515700$ Al $0.80964200-0.53346300-0.01245900$ B -2.01501500 -0.36149800 0.08621800 H - $1.09690300-1.05024400-0.30267900$ H -3.04564000 -0.71540400 -0.39231600 H -2.01724300 -0.03840000 1.22665000 H -2.09246500 $0.71835800-0.75765600$ H $1.15338500-1.187659001 .37777200$ H 1.17870400 -1.15919300 - 1.40489700 H - $1.419091000 .87235300-0.35347700$ H 0.645073001 .755330000 .71498400

\subsection{Coordinates of tR1-tR12}

tR1 reactant:

O $1.755149000 .16005700-0.15527500$

B -1.39033800 0.71074800-0.09080900
H 2.388637000 .378167000 .53871000 H $1.389018001 .00276400-0.46151100$ H - 1.038029001 .370574000 .84770200 H $-2.562726000 .47488900-0.15045000$ H $-0.884935001 .02470800-1.14373400$ H $-0.97956000-1.43490500-0.40680000$ O $-0.70873800-0.784840000 .25537000$ H $0.26809900-0.664534000 .17948500$

tR1 TS:

O -2.30439000 0.07576400 0.04987700 B $1.844818000 .48594400-0.08425100$ H - $1.34292700-0.037608000 .02395700$ H - 2.47301100 0.93811700-0.34340300 H $1.481746001 .61261100-0.09943300$ H $2.700820000 .08515500-0.80147800$ H 2.186141000 .190749001 .14677500 Н $0.75890800-1.32763400-0.52687000$ O $0.56545600-0.51553900-0.03513600$ H $1.37570300-0.372905000 .90377400$

tR1 product: O 2.10579700 $0.02936000-0.21282600$ B -1.69143800 0.50689300 0.57683700 H $1.14114500-0.04492100-0.22248900$ H $2.355444000 .26745700-1.11159600$ $\mathrm{H}-1.625219001 .569742000 .05914800$ $\mathrm{H}-2.487628000 .233660001 .41663100$ $\mathrm{H}-0.390355000 .16048700-3.38319400$ $\mathrm{H}-0.87694000-1.279204000 .61056700$ O $-0.78949800-0.433767000 .15082700$ H $-0.53009300-0.02618900-2.68636800$

tR2 reactant:

O - $1.59905100-0.59474600-0.46860200$ B $1.147361001 .02505000-0.28157200$ H - 2.45477900 -0.31194000 -0.12473500 $\mathrm{H}-1.353127000 .05184800-1.14641000$ H 2.29731300 $1.23991100-0.02637200$ H 0.371626001 .879975000 .04650500 H - $0.15492700-0.533003000 .48744500$ O $0.74546400-0.231803000 .75765900$ H $1.35475600-0.972184000 .63519400$ Н $0.952613000 .56585700-1.38332900$

tR2 TS:

O 1.945804000 .125887000 .08717200

B - $1.568196000 .56989100-0.09911500$ H $2.68018000-0.35518900-0.31195400$ H $2.034893001 .03980400-0.20903600$ $\mathrm{H}-2.537161000 .50890000-0.78215600$ $\mathrm{H}-0.897449001 .55620300-0.08376800$ H $0.21490000-0.54076400-0.01821400$ O $-0.73135100-0.76613800-0.10833800$ 
H - $1.37927000-0.338470000 .91363100$ H -1.99073100 0.40207200 1.15640200

tR2 product:

O - $1.85775800-0.64412300-0.38172800$

B 1.055683001 .220674000 .39815300

H -2.29137900 - $1.43648000-0.04414200$

H -2.50159300 $0.06892800-0.29769200$

H 2.157648001 .587726000 .64909000

H 0.130553001 .977830000 .35942100

H -0.05595300 -0.31914600 -0.04485000

O $0.87248400-0.101535000 .15551900$

H $3.03381900-1.50634200-0.57957200$

H $3.63180100-1.87280300-0.80150900$

tR3 reactant:

O -0.580277000 .407380000 .86856700$

Al 1.296867000 .467283000 .12860200

$\mathrm{H}-0.656731000 .076094001 .77239000$

H - $1.17813700-0.122526000 .28374600$

H $1.05983800-0.58984500-1.06591300$

H $1.483297001 .97292600-0.32760200$

H $2.07551400-0.070584001 .40645600$

O - $1.59842800-1.14048300-1.01765300$

$\mathrm{H}-0.71010100-1.18934700-1.41202800$

H -2.21584500 - $0.95198000-1.73388300$

\section{tR3 TS:}

O -0.042813 0.578412-0.017020

Al $1.698699-0.283099-0.046964$

H 0.6626900 .8494530 .926531

H $-0.2200261 .337998-0.593418$

H $1.582905-1.8234970 .223018$

H $2.6713700 .414147-1.065407$

H 1.5782460 .6378391 .427698

O -2.867907 -0.150573-0.058931

H - $1.912809-0.1283650 .097441$

H -3.159698 -1.030004 0.202283

tR3 product:

O -0.39062700 0.311947000.59302800

Al 1.283705000 .556862000 .16732600

H $1.87979300-0.348158003 .10230600$

H -0.926348001 .030320000 .94797000$

H $2.02395500-0.71266400-0.36673400$

H 1.909142001 .987010000 .34672700

H 2.480071000 .075111003 .06112000

O $-2.00775600-1.19924100-1.23099300$

H $-1.48376700-0.76874800-0.53519200$

H -2.18618600 -2.08920700 -0.91089500

tR4 reactant:

O $-0.60684100-0.90124600-0.27678500$

Al $1.33085900-0.37540100-0.06795900$
H $-0.89271400-1.646282000 .26698700$

H - $1.20632200-0.13250600-0.10383200$

H $1.90610300-0.43038200-1.54305600$

H 1.060526001 .105256000 .51096100

H $1.76658100-1.480637000 .98896900$

O - 1.628209001 .452300000 .36194100

H -0.720986001 .767093000 .51973000$

H -2.07113600 2.12208100-0.17184800

tR4 TS:

O $0.093427-0.856724-0.158842$

Al $1.5375100 .339290-0.034613$

Н $0.713144-0.9379250 .921964$

H $-0.824632-0.528384-0.088758$

H $2.782327-0.008709-0.929367$

H 1.0858771 .8189560 .286530

H $1.536804-0.6260221 .451500$

O -2.5157170 .2112620 .053425$

H -2.6363011 .1673480 .096024$

H -3.266524 - $0.132345-0.444580$

tR4 product:

O -0.27784200 -0.43202400 -0.46898900

Al $1.41388400-0.61238300-0.21574200$

H $0.42528700-2.202749002 .34307700$

H $-0.819715000 .29660200-0.12998400$

H $2.07617600-1.89048200-0.84364600$

H 2.240987000 .470402000 .57927100

H $1.09697900-2.200355002 .64236300$

O -2.09339500 1.589629000 .50176200

$\mathrm{H}-2.292493002 .35830800-0.04524500$

H -2.94654600 1.190982000 .70988600

tR5, Step 1 reactant:

O -0.44557100 - 1.570297000 .12545700

B $1.300179001 .09407900-0.17215000$

B 2.419756000 .432578000 .98196600

H 2.365317001 .599566000 .38858500

H $3.37696900-0.145786000 .59144800$

H 2.211568000 .582856002 .13865100

H $1.35263100-0.066467000 .41519600$

H 0.349827001 .686504000 .22518200

H $1.500440000 .96239500-1.33257500$

H $-0.79002900-2.287572000 .66790400$

H - $1.21985700-1.05644700-0.15943800$

O $-2.400708000 .34160400-0.72820300$

H -2.69905600 $0.38922800-1.64396300$

H - $1.911229001 .15903700-0.57124700$

tR5, Step 1 TS:

O $0.1245760 .872071-0.227941$

B -0.864642 -0.616135 -0.326231

B -2.568166 -0.0720380 .292695$

H -2.012141 -1.183714 0.188848 
H -3.597056 -0.265481-0.276815 H -2.575782 0.1939831 .459302 H - $1.9511900 .701527-0.399869$ $\mathrm{H}-0.149913-1.2653990 .393273$ H $-0.838062-0.866292-1.486846$ H -0.1594471 .4020320 .532448$ H $1.0576530 .593236-0.064315$ O $2.580065-0.1979170 .213280$ H $3.334526-0.117898-0.382653$ H $2.418325-1.1443610 .321596$

tR5, Step 1 product: O $0.13300100-0.83409600-0.54021800$ B $1.187029000 .29704500-0.81502200$ B 1.524866000 .348605001 .45106600 H 1.484470000 .925302000 .25700000 H 2.350404001 .063898001 .93521000 H 0.392926000 .509033001 .83610100 H $1.86669100-0.801802001 .33704800$ H $0.613385001 .14239300-1.43367500$ H $2.16295500-0.19321000-1.27802500$ H $0.43430700-1.333510000 .24138100$ H $-0.76869500-0.45810500-0.34347600$ O $-2.230720000 .23771100-0.04934400$ $\mathrm{H}-2.696307000 .65016000-0.78779900$ H -2.17209100 0.907937000 .64498100

tR5, Step 2 reactant: O $-0.67795400-0.19304400-0.85224200$ B -0.19509700 1.29359600 -0.73109800 B $1.911500000 .46528000-0.31772700$ H $0.957661001 .37085400-0.17999900$ H $2.840167001 .20259000-0.17204100$ H $1.68734500-0.277434000 .60756800$ H $1.83876400-0.04671400-1.40529500$ H -0.883931001 .800300000 .10137800$ $\mathrm{H}-0.149179001 .77016200-1.81636500$ H $0.01409900-0.69642100-1.31730700$ H -0.79169200 -0.60499800 0.05237200 O $-0.76917900-1.185609001 .57305600$ H - $1.40683800-0.877881002 .22925100$ H $0.11095900-0.973892001 .91777900$

tR5, Step 2 TS:

O $-0.173617-0.824108-0.647512$

B - $0.932415-1.0542670 .572671$

B - 1.5937561 .2436450 .086192

$\mathrm{H}-1.7592100 .2127990 .760540$

H -2.593756 1.8916550 .075027

H -0.5672301 .7917200 .349669$

H - $1.5952130 .858386-1.165591$

H $-0.331225-1.0323791 .599908$

$\mathrm{H}-1.891022-1.7417400 .430094$

H $-0.9525040 .250255-0.981962$
H $0.727214-0.489416-0.452679$

O 2.2420800 .3603810 .030127

H $2.984947-0.1017790 .436357$

H 2.0611511 .1234290 .593401

tR5, Step 2 product:

O $-0.598344000 .28679700-0.81284000$

B -1.54832900 1.21836000-1.10025500 B 2.52624000 -0.09731300 0.01674900 H 2.191704000 .774757000 .75672600 H $3.64145600-0.01486800-0.40233500$ H $2.14715300-1.209858000 .22914700$ H $2.190058000 .22496200-1.28758300$ H - 2.47880500 $1.37660300-0.37129500$ $\mathrm{H}-1.402613001 .85374800-2.09367200$ H $1.491227000 .21630000-0.91596600$ H $-0.75444200-0.194350000 .02572100$ O - $0.49598000-1.120775001 .55888400$ $\mathrm{H}-0.80812800-0.858444002 .43268700$ H $0.46756700-1.179128001 .62235200$

tR6, Step 1 reactant: O $1.80772600-0.21459800-0.18551900$ Al -0.18401900 -0.20316600-0.75856400 Al - $1.926748001 .68705100-0.11100100$ H $2.44953100-0.28507900-0.90355600$ H $2.01274400-0.895704000 .49776200$ H $-0.52030400-1.456301000 .16532600$ $\mathrm{H}-1.985275000 .26927900-0.97856100$ H $0.17885700-0.15322700-2.29438500$ $\mathrm{H}-2.021510002 .96667300-1.02657700$ H -2.60696900 1.546644001 .30574200 H -0.253889001 .298716000 .08501900$ O $1.75444000-2.207983001 .59737200$ H $1.92123000-2.234269002 .54677600$ Н $0.80098200-2.335251001 .47533600$

tR6, Step 1 TS:

O $0.894937-0.6899790 .623577$

Al -0.1139340.9161540.120779

Al $-2.513710-0.374416-0.243510$

H $0.641970-1.2932981 .333105$

H $1.858121-0.7303690 .391064$ H $1.0533721 .309348-0.914795$ H - $1.3970650 .725349-0.985508$ H -0.394030 1.9211961 .298523 $\mathrm{H}-3.8209480 .4517470 .065991$ H -2.483782 -1.674690 -1.144207 H - $1.465741-0.4488621 .014984$ O $3.283833-0.203661-0.334021$ H $3.844198-0.689446-0.950715$ H $2.8931120 .535553-0.829383$ 
tR6, Step 1 product:

O $1.27449500-0.25567100-0.59480600$ Al -0.39910000 -0.83956900 - 1.36089400 $\mathrm{Al}-1.113540001 .559794000 .49690000$ H $1.305354000 .72138200-0.53001000$ H $1.42624900-0.607906000 .33076700$ H - $0.60433100-2.26697400-0.72522000$ H - $1.425616000 .23538000-0.59234000$ $\mathrm{H}-0.36071000-0.48381200-2.88988200$ H - 2.429217002 .426396000 .42734900 $\mathrm{H}-0.782579000 .735909001 .83854300$ H $0.240431002 .12953800-0.17243700$ O $1.33192900-0.990435001 .91900600$ H $0.55257900-0.491331002 .22275900$ H 1.20847500 -1.90386600 2.20537600

tR6, Step 2 reactant: O $0.963355000 .69172700-0.81389700$ Al $0.96650900-1.22323800-0.56245600$ Al - $1.94838700-0.309186000 .07666300$ H $0.135275000 .98427100-1.24643700$ H 1.030276001 .205781000 .04366900 H $1.91692600-1.421377000 .67849100$ H $-0.61440700-1.42334500-0.05203900$ H $1.11814800-1.87300600-1.98401800$ H -3.22111500 - 1.240438000 .08181600 $\mathrm{H}-1.532774000 .456790001 .42930500$ H - $1.647355000 .60626900-1.21752900$ O 0.785695001 .861704001 .52109400 H - 0.064695001 .477296001 .80185600 H 1.407905001 .729091002 .24682400

tR6, Step 2 TS:

O - $0.5576780 .332452-0.858871$ Al 0.2175531 .5583750 .236447 Al $1.873392-1.0341390 .155019$ H $0.420164-0.591828-1.224806$ H - $1.386189-0.098293-0.569712$ H - 0.4531211 .6083171 .668383 H 1.6907830 .5892450 .450844 H $0.7897422 .825920-0.502944$ H $3.369227-1.353059-0.209980$ H $0.953440-1.8770091 .112739$ H $1.091780-1.112970-1.425353$ O $-2.831808-0.9572330 .173280$ H -2.794916 - 1.0704871 .130974 H -3.747303 -0.736653 -0.034476

tR6, Step 2 product: O $0.060768000 .36102200-0.30861600$ Al $0.92567500-1.28463900-0.52739600$ $\mathrm{Al}-1.61571100-0.44403800-0.09453800$ $\mathrm{H}-0.973776002 .69401800-1.84842300$ H 0.417525001 .057747000 .27787600
H $1.74256600-1.666796000 .76941300$ H - $0.69644500-1.90126900-0.39522200$ H $1.44734800-1.54346100-1.98916700$ H - $2.57991000-0.21254000-1.31537700$ H $-2.08099900-0.435933001 .41230800$ H - $1.230426003 .37672800-1.93795600$ O 1.152995002 .167170001 .45220200 H 1.505252001 .826427002 .28324600 H 1.659222002 .961053001 .24420000

tR7 reactant: O 0.031352000 .171270000 .59080100 $\mathrm{Al}-1.86333800-0.656539000 .09059000$ Al $1.88675100-0.792513000 .14301800$ H 0.038005000 .415943001 .53244000 H 0.051324001 .039396000 .03711100 H $1.50460600-2.317640000 .26188300$ H $2.00430700-0.09917700-1.28752200$ H $2.66036500-0.093558001 .34492300$ H -2.47240700 $0.76045500-0.34409500$ H - $1.44180300-1.61993800-1.08616500$ $\mathrm{H}-2.21728100-1.188381001 .53883200$ O $-0.053972002 .19624400-0.92445100$ H $0.558161002 .11678000-1.66988600$ H - $0.953104002 .20604200-1.28639600$

tR7 TS:

O 0.1397530 .0904020 .230209

Al -1.084141 -1.512224-0.072860

Al 2.121894 0.243002 -0.115592

H 0.8158490 .1672031 .185828 H - 0.4239350 .9145320 .111633 H $2.698454-1.112838-0.638053$ H $2.3979071 .689330-0.659166$ H 1.8527180 .2421031 .575906 H - $2.459795-0.7302090 .226166$ H - $0.798477-1.807490-1.606565$ H - $0.547504-2.5014751 .042189$ O - 1.7247791 .8988130 .008623 $\mathrm{H}-1.9194392 .499648-0.720843$ H - 2.4263481 .2253720 .022119

tR7 product: O $0.21979500-0.06083100-0.18266500$ $\mathrm{Al}-1.64858200-0.580378000 .20106500$ Al $1.71427200-0.890203000 .27812600$ H $1.05195900-0.197814002 .79780000$ H $0.240199000 .86790500-0.52316100$ H $1.54988600-2.382621000 .69332300$ H $3.02778600-0.059978000 .09516300$ H 1.751840000 .037129002 .78832700 H - $2.326548000 .87088800-0.00438200$ H - $2.00099900-1.66148500-0.91024900$ H - $1.50826000-1.074962001 .71054200$ 
O $-0.340350002 .44707600-0.89253100$ H $-0.371228002 .86643100-1.76038900$ H - $1.259996002 .23351400-0.65176700$

tR8 reactant: O $-2.28250300-0.144058000 .73611200$ B 0.668869001 .355906001 .16611400 Al $1.32861300-0.06504600-0.43110900$ H -3.07985300 0.380074000 .59443000 $\mathrm{H}-1.729817000 .352976001 .36107400$ H 0.074344000 .766803002 .03376700 H 1.807039000 .878547001 .08841400 H 0.782390002 .535678001 .28755800 H $1.950232000 .65768500-1.68182400$ H $1.88539200-1.441855000 .12100200$ H 0.093827001 .191909000 .08448000 H - $1.21889600-0.61203100-0.47146900$ H $-0.39932400-1.82697300-1.06651000$ O $-0.42951100-0.86113100-1.02265800$

tR8 TS:

O $1.270664000 .77061600-0.79436700$ B -0.90856800 1.26619300 1.03824700 Al -2.43323400 -0.63879800-0.16113200 H $0.629234000 .28699300-1.33436500$ H $1.656488001 .44062300-1.37256000$ $\mathrm{H}-1.269363002 .141589000 .33710000$ H - 1.672706000 .366471001 .30909300 H 0.088720001 .269233001 .66322100 H -2.45850800 -1.99100100 0.64538300 H -3.73314500 $0.19935900-0.45622100$ H - $1.12996200-0.29947100-1.01682500$ H $2.70055700-0.30408200-0.01629300$ H $3.38725300-0.874714001 .23833900$ O $3.47637600-0.803316000 .28269300$

tR8 product: O - $1.15690600-0.017740000 .54678400$ B -0.23488000 1.27072600 0.55711900 Al $1.98374500-0.242425000 .61984300$ $\mathrm{H}-0.60124900-0.762027000 .88228200$ $\mathrm{H}-1.48553000-0.24124600-0.36030000$ H -0.026867001 .544717001 .69699600$ H $0.805618000 .95585000-0.06069600$ H $-0.770008002 .09266900-0.12249000$ H $2.52994700-0.67147800-0.80808300$ H 2.895304000 .531483001 .64737200 H $0.88753600-1.273626001 .22179700$ H - $1.39605500-0.43113700-2.64882900$ H $-2.84926500-0.13476800-2.21683800$ O -2.02710500 - $0.54943100-1.92741000$

tR9 reactant: O $0.70282200-0.58297900-0.71679200$
B -1.41092500 0.35777100 2.03262100 Al - $1.11549500-0.836644000 .17097400$ H $1.34237600-1.28001400-0.52343300$ H $1.194736000 .25195200-0.88537700$ H - 1.984892001 .405130002 .02102400 H -2.19144700 -0.51624000 1.62676900 H $-0.91942000-0.015380003 .05587900$ $\mathrm{H}-0.77822300-2.349171000 .48671700$ H -2.02939000 - $0.28247600-0.98569100$ H - 0.479350000 .467624001 .19979900 H 2.18085700 2.08082600 -2.03323200 H $2.318330002 .30233500-0.51218900$ O $2.234864001 .61795700-1.18807300$

tR9 TS:

O - $1.111728-0.428034-0.362307$

B $0.8539391 .387073-0.131647$

Al $2.800277-0.3553130 .122881$

H $-0.820547-1.2686410 .008749$

H - $2.054578-0.344013-0.143507$

H 0.6028381 .4967661 .014124

H $2.0200481 .247730-0.459301$

H $0.1601061 .718577-1.021502$

H $3.622485-0.625990-1.197228$

H 3.5149320 .1029281 .453451

H $1.327504-0.9491700 .237837$

H -4.207298 0.5949330 .826597

H -4.526337 -0.051749-0.532465

O $-3.927327-0.1016730 .221560$

tR9 product:

O $0.89447600-0.09118300-0.12482200$

B -0.09583700 0.57606500 0.93001200

Al - $1.91755400-1.312224000 .44714700$ H $0.32639700-0.60127000-0.75127900$ H $1.434299000 .58278300-0.60735600$ $\mathrm{H}-0.832907001 .321987000 .35321000$ $\mathrm{H}-0.67362100-0.398553001 .42954900$ H 0.576635001 .044939001 .79617200 $\mathrm{H}-1.77566800-2.643461001 .29192700$ $\mathrm{H}-3.24417900-0.458629000 .48586400$ H - $1.09393400-1.31600300-0.94973100$ H $2.066109002 .68545800-1.28539400$ H $3.330813001 .82559500-1.06625700$ O $2.398998001 .77952700-1.31348800$

tR10 reactant:

O -0.69162000 -0.55750600 0.21178000 B - 0.335468000 .983012000 .40940900 Al 2.26197100 0.38246000 0.55758200 H 0.636530000 .949951001 .17640200 H $2.886921001 .58187800-0.25522900$ H $2.80438400-0.005164001 .99325700$ H $1.70648500-0.87188800-0.30749500$ 
H - 1.229522001 .476496001 .02516000 H - $0.082535001 .46311500-0.65736800$ H $0.12463800-0.98226800-0.14847300$ $\mathrm{H}-1.45350500-0.69053400-0.40469300$ O $-2.81240700-0.86724600-1.39836000$ H $-3.03033400-0.13425500-1.98782800$ H -3.64459800 -1.15297000 -1.00176400

\section{tR10 TS:}

O -0.532865 0.1457720.457561

B $0.1784441 .348061-0.202997$ Al 2.220436 -0.410721 -0.091824 H $1.3083800 .916388-0.661693$ H $3.233508-0.785172-1.229071$ H $2.514404-0.1910901 .435149$ H $0.851435-1.442116-0.176699$ H 0.4253952 .1973390 .596073 H -0.384908 $1.647376-1.210046$ H $0.191612-0.8205130 .150062$ H -0.5652860 .2350761 .422867$ O -3.352064 -0.517978-0.112858 $\mathrm{H}-2.440587-0.194433-0.105070$ H -3.812401 $0.013855-0.770498$

tR10 product: O $0.005326000 .40528000-0.43139200$ B -0.34723300 1.45259600 0.67833300 Al $1.46214800-0.001593000 .62347900$ H 0.652322001 .318243001 .48675200 H 2.821642000 .553022000 .07352100 H $1.27772900-1.302223001 .48695700$ H $1.18808000-2.48768800-2.07897100$ H - 1.321263001 .122654001 .29125000 H -0.288714002 .568270000 .26152500$ H $0.50908200-2.50841600-1.79845800$ $\mathrm{H}-0.73355500-0.21025800-0.61677700$ O $-2.14825100-1.20145200-0.96933300$ H -2.67442500 -0.94976700 - 1.73819700 H - $2.77271300-1.29326100-0.23887800$

tR11 reactant:

O - $1.24249000-0.23862100-0.82190200$ B -0.82218800 1.27995600 -0.69396800 Al $1.709360000 .32008600-0.17848300$ H $0.251667001 .32814700-0.04486800$ H $2.868919001 .37131100-0.35218000$ H $1.48921000-0.391724001 .24540300$ H $1.28833700-0.63914500-1.39444000$ H - 1.630395001 .798179000 .01254100 H -0.66921400 $1.70345300-1.79532000$ H - $0.54798000-0.71951100-1.31475700$ H - $1.32391200-0.673249000 .07677600$ O - $1.07348900-1.289119001 .56719100$ H -0.14753200 -1.06524200 1.77190500
H -1.60447200 -1.01725200 2.32580100

tR11 TS:

O 0.3976921 .0238640 .643008

B -0.289280 1.496301 -0.588960

Al - $1.567676-0.767560-0.070049$

H $-1.3671850 .699052-0.820621$

H -3.084682 -1.178894-0.050970

H $-0.374630-1.741235-0.409642$

H - $1.263277-0.1853701 .568844$

H $0.3525851 .351922-1.589040$

H -0.871993 2.525937 -0.428194

H -0.5658100 .2745811 .302477$

H 1.1966400 .4928920 .444399

O $2.434427-0.746867-0.028127$

H $1.961563-1.447451-0.496157$

H $3.186016-0.510636-0.584703$

tR11 product:

O $-0.33415700-0.16734700-0.57523000$

B -0.76165700 1.33996900 -0.57407400

Al $1.368207000 .49063400-0.27476800$ H $0.370541001 .94237300-0.36828700$ H $2.327059000 .47621200-1.51288500$ H 1.791680000 .306482001 .23184000

H $0.53274300-2.72278900-2.80431100$ H -1.44589800 1.598863000 .37151700

H - $1.122830001 .68345900-1.65641600$ H $0.22226800-2.14313400-2.47574000$ H $-0.75685900-0.663332000 .15859900$ O $-1.36632000-1.450168001 .61254500$ H $-0.92706800-1.180533002 .42892200$ H -2.31333900 -1.39932400 1.79101100

tR12 reactant:

O $0.570359000 .94246200-0.81904400$ Al $0.19990200-1.01116200-0.39225900$ B - $1.95726800-1.54545500-0.29559200$ H - $1.02896000-2.310261000 .01117700$ H -2.54968300 -1.24274400 0.69678700 H $-1.45700800-0.49331600-0.76219800$ H - 2.56026600 -2.04012600 - 1.19835300 H $0.76731800-0.827912001 .08226300$ H $0.93823600-1.67899300-1.60926400$ H $0.110679001 .40853700-1.52868200$ H $0.606560001 .51411900-0.01233800$ O 0.640689001 .957013001 .63314700 H 1.284998002 .521353002 .07640600 H 0.696992001 .085738002 .05435000

tR12 TS:

O $0.162897-0.107886-0.830827$

Al -0.954153 -1.061752 0.183601

B -1.735654 1.5488050.293162 
H -2.178909 0.4072370 .264963

H -2.635360 2.316483 0.153802

H -0.9394531 .7730961 .145989$

H - $1.2359961 .826753-0.906439$

H -0.492794 -1.349463 1.664679

H - $1.979957-1.967120-0.591771$

H $-0.7087651 .181270-0.756306$

H $1.0799480 .030869-0.533171$

O 2.7926770 .3119600 .182239

H $3.599865-0.006980-0.237833$

H 2.9290880 .2140151 .132175

tR12 product:

O $0.181179000 .53708400-0.64662900$
Al $0.61340500-1.16681600-0.49082200$

B -2.18268400 -1.03752500 -0.29435600

H $-1.28108100-1.74328800-0.70071900$

H -3.23202600 - $1.41986700-0.70798600$

H -2.12840400 -0.69090900 0.83905200

H -2.28862500 $0.02720500-1.13162100$

H $1.01733800-1.699229000 .93957000$

H $0.99606300-1.90970400-1.82322600$

H - $1.555538000 .17192700-0.81620100$

H 0.355811001 .145913000 .09069500

O 0.667240002 .229128001 .62809200

H 1.263679002 .986493001 .60869400

H 0.808913001 .801487002 .48102100 